\title{
Using motor tasks to quantitatively judge 3-D surface curvatures
}

\author{
HIROSHI WATANABE \\ ATR Human Information Processing Laboratories, Kyoto, Japan \\ and Osaka National Research Institute, Osaka, Japan \\ FRANK E. POLLICK \\ ATR Human Information Processing Laboratories, Kyoto, Japan \\ and University of Glasgow, Glasgow, Scotland \\ JAN J. KOENDERINK \\ Helmholtz Institute, Universiteit Utrecht, Utrecht, The Netherlands \\ and \\ MITSUO KAWATO \\ ATR Human Information Processing Laboratories, Kyoto, Japan
}

\begin{abstract}
The primary objective of this study was to quantitatively investigate the human perception of surface curvature by using virtual surfaces and motor tasks along with data analysis methods to estimate surface curvature from drawing movements. Three psychophysical experiments were conducted. In Experiment 1, we looked at subjects' sensitivity to the curvature of a curve lying on a surface and changes in the curvature as defined by Euler's formula, which relates maximum and minimum principal curvatures and their directions. Regardless of direction and surface shape (elliptic and hyperbolic), subjects could report the curvature of a curve lying on a surface through a drawing task. In addition, multiple curves drawn by subjects were used to reconstruct the surface. These reconstructed surfaces could be better accounted for by analysis that treated the drawing data as a set of curvatures rather than as a set of depths. A pointing task was utilized in Experiment 2, and subjects could report principal curvature directions of a surface rather precisely and consistently when the difference between principal curvatures was sufficiently large, but performance was poor for the direction of zero curvature (asymptotic direction) on a hyperbolic surface. In Experiment 3, it was discovered that sensitivity to the sign of curvature was different for perceptual judgments and motor responses, and there was also a difference for that of a curve itself and the same curve embedded in a surface. These findings suggest that humans are sensitive to relative changes in curvature and are able to comprehend quantitative surface curvature for some motor tasks.
\end{abstract}

Surfaces are rich in visual information that can potentially be used to perceive the underlying structure of a surface (Gibson, 1979). When addressing a perceived surface structure, it must be done from two aspects: its qualitative features (Todd \& Reichel, 1989) and quantitative features (Koenderink \& van Doorn, 1991). In some cases, describing a surface in a qualitative manner is sufficient for understanding its appearance. For other tasks, however, it seems that quantitative surface aspects should be utilized. Dealing with the surface geometry in the sense of Euclidean metrics, depth, orientation, and curvature can be considered as primitive descriptors of a three-dimensional

This work was supported by an HFSP grant to J.J.K. and M.K. Correspondence should be addressed to $H$. Watanabe, Osaka National Research Institute, 1-8-31 Midorigaoka, Ikeda, Osaka, 563, Japan (e-mail: watanabe@onri.go.jp).

-Accepted by previous editor, Myron L. Braunstein
(3-D) surface geometry (Erens, Kappers, \& Koenderink, 1993). Many studies of computer vision have shown that these descriptors are adequate for achieving high performance with a machine (Horn, 1986) and thus are at least a plausible description for aspects of human vision. In the human vision system, is this quantitative geometrical information utilized to understand a 3-D structure?

Many psychophysical approaches have recently been used to study the sensitivity and precision of the human vision system for metric descriptors. Most of these behavioral studies have addressed the relationship between surface perception and visual cues (e.g., horizontal disparity, texture, shading, motion, etc.) and have looked at the accuracy and stability of the responses for a metric surface geometry by controlling visual cues attached to virtual surfaces. It is known that when humans are asked to perform such tasks, although their responses are self-consistent, there is frequently systematic biases to their data. One example of this is the tendency when presented a shaded 


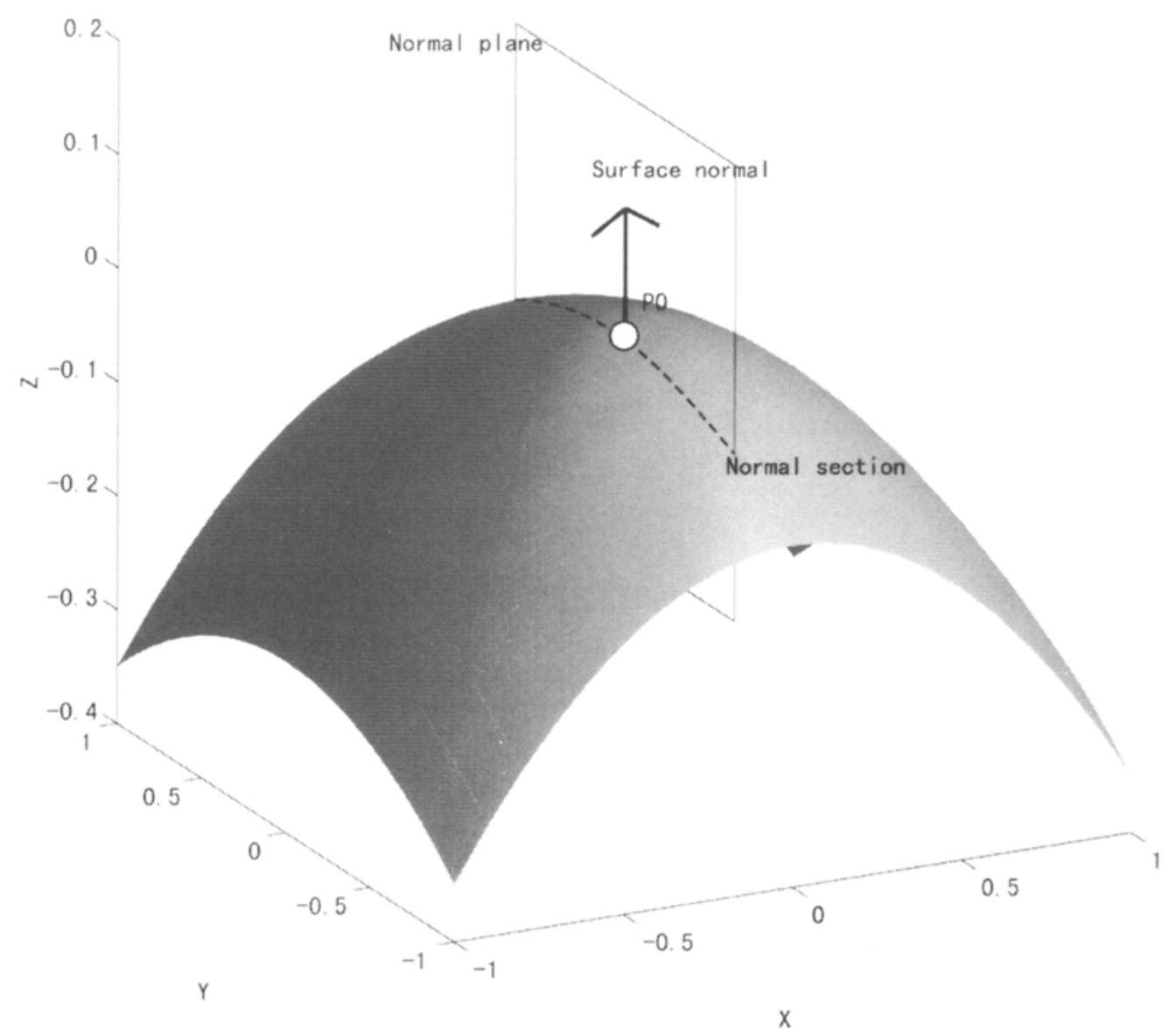

Figure 1. Surface, surface normal, and normal plane. A normal plane contains a surface normal at $\boldsymbol{P}_{\mathbf{0}}$. An intersection between the surface and the normal plane is called normal section, and its curvature is called normal curvature. The changing of normal curvature with the rotating of the normal plane around the surface normal could be described by Euler's formula (Equation 1).

image to judge the surface to be flatter than the shape being simulated (Bülthoff \& Mallot, 1988; Mingolla \& Todd, 1986; Pollick, Watanabe, \& Kawato, 1996). Humans do not always report a physical entity as it is, and reported surface structures frequently agree between and/or within subjects (Koenderink, van Doorn, \& Kappers, 1992; Pollick et al., 1996). Such findings suggest that the human vision system codes and reconstructs surface geometry; in other words, these descriptions of surface geometry are consistently represented. Visual psychophysics has primarily studied depth (Bülthoff \& Mallot, 1988) and orientation (Koenderink et al., 1992; Norman, Todd, \& Phillips, 1995; Pollick et al., 1996) and, to a lesser extent, curvature (Johnston \& Passmore, 1993; Kappers, Koenderink, \& Lichtenegger, 1994; Schwartz, 1994). Little evidence has suggested the ability to judge quantitative surface curvature by humans.

The curvature of a surface does not mathematically change if the surface is translated in depth or rotated in orientation, so this plays an important role in describing local surface shape. Here, we summarize the concept for the measurement of 3-D surface curvature (for a more general description, see the Appendix). One of the most primary elements of surface curvature is curvature of a curve on the surface. The plane that contains the surface normal at a point $P_{0}$ is called the normal plane, and the normal curvature is defined as the curvature of the curve at the intersection between the surface and a normal plane (Figure 1).

The normal curvature changes with the rotating of the normal plane around the surface normal at $P_{0}$, and its changing could be described by the following equation, called Euler's formula (de Vries, Kappers, \& Koenderink, 1994; Lipshutz, 1969; O'Neill, 1966):

$$
\kappa_{N}=\kappa_{\max } \cos ^{2} \alpha+\kappa_{\min } \sin ^{2} \alpha,
$$

where $\kappa_{\max }$ and $\kappa_{\min }$ are the maximum and the minimum principal curvature, respectively, which have the maximum and minimum curvature within the infinitely large family of the normal curvature. Also, the direction of the normal plane that gives $\kappa_{\max }$ and $\kappa_{\min }$ is called the principal direction. The two directions of principal curvature are always orthogonal, and $\alpha$ is the angle between $\kappa_{\max }$ and the curves in question (Figure 2). 


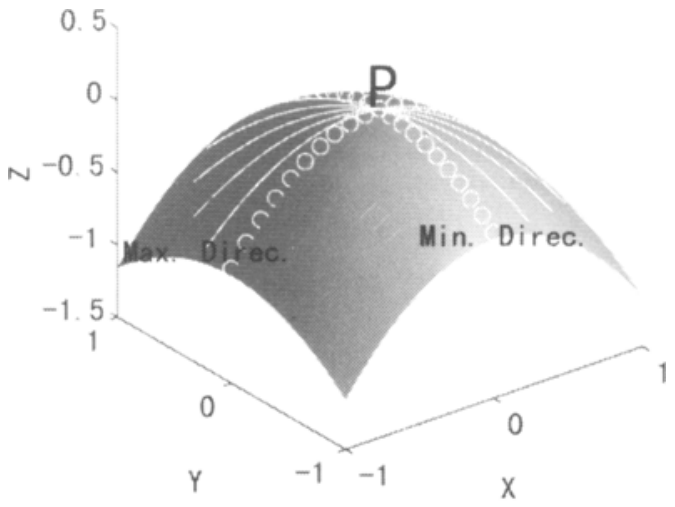

\section{Elliptic Surface}

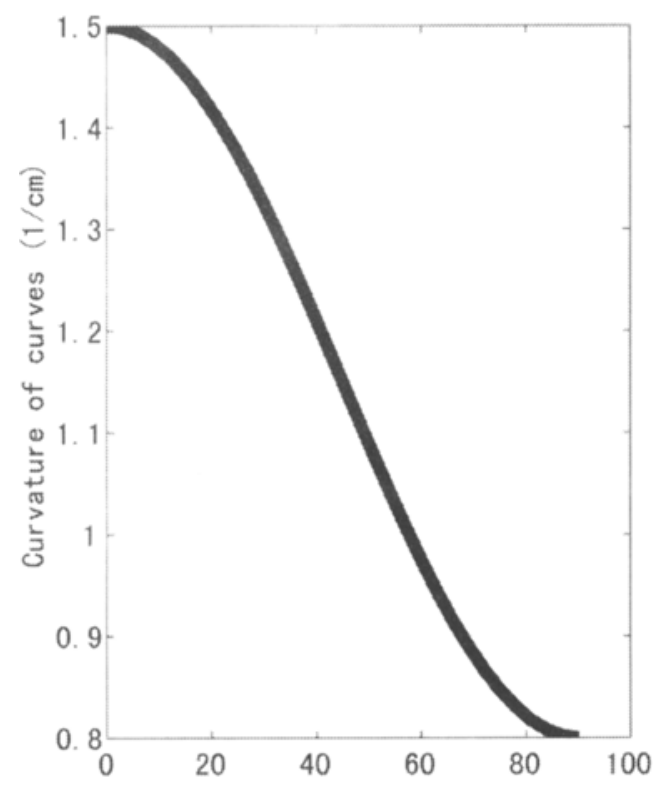

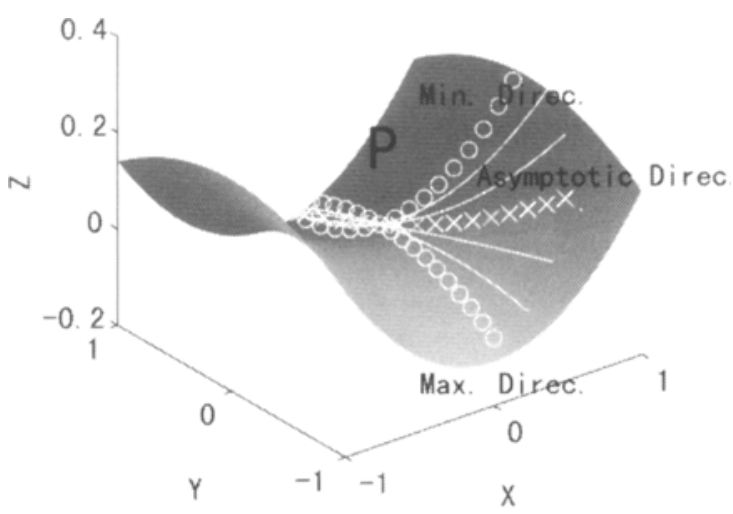

Hyperbolic Surface

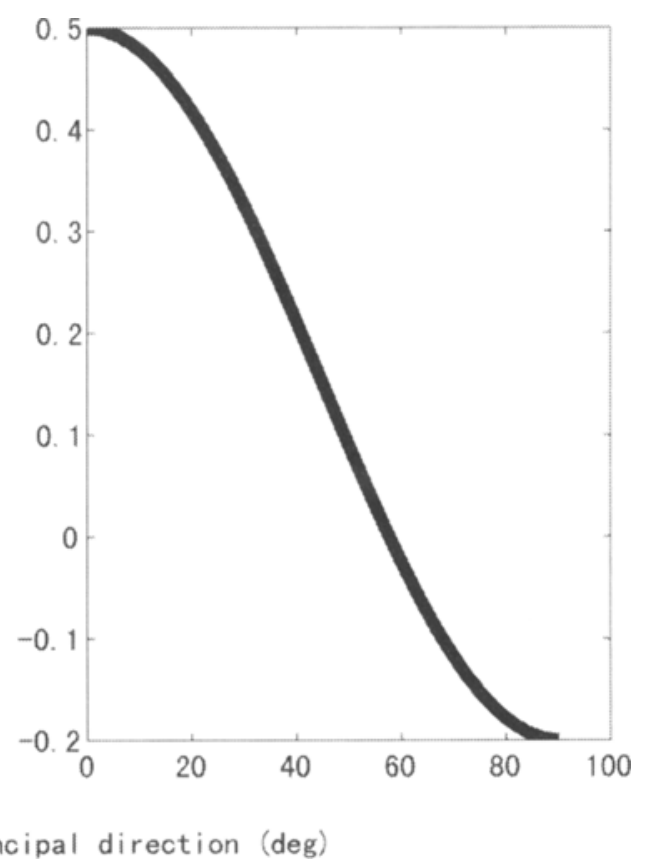

Figure 2. A family of curves lying on the elliptic and hyperbolic surfaces (upper) and changes of the curvature of curves at $\boldsymbol{P}$ (lower). For the asymptotic direction on the hyperbolic surface, the curvature of a curve becomes zero.

In contrast to depth and orientation, curvature has two advantages for describing local surface shape. First, curvature has both a qualitative function for describing surface shape and a quantitative function that measures magnitude of surface curving. A local shape, for example, can be classified into two groups by using the sign of product of the maximum and minimum principal curvatures (Gaussian curvature, $K$ ). When $K$ is positive, the local shape is characterized as elliptic; when $K$ is negative, it is characterized as hyperbolic. The boundary between these two categories, when $K$ is zero, is characterized as parabolic. In addition to this qualitative aspect, we can compare the size of objects, such as two spheres, by using the radius of the principal curvatures. Second, the curvature description of the surface does not depend on the viewpoint. Regardless of viewing direction and the distance between observer and object, classification of the surface shape by Gaussian curvature and comparison of principal curvature radii are achieved. Similar to other perceptual situations, we should notice that there exists an adequate range of curvature to which humans are sensitive (Phillips \& Todd, 1996). 
Some psychophysical evidence suggests that humans utilize the principal curvature for perceiving surface geometry. Stevens (1981) showed that human subjects could reconstruct the surface geometry using the curves depicted on the surface on the assumption that they agree with the principal curvature locally. Kappers et al. (1994) studied haptic sensation for real surfaces. When subjects evaluated the surface shape using only haptic sensation, it was observed that the principal curvature and its direction could be informative about surface shape. Koenderink (1990) proposed a shape index and curvedness that combine the principal curvatures; these scales independently describe surface shape and the amount of curvature. He and his colleagues performed psychophysical experiments of surface perception using these scales, and the results suggest that they can be utilize with intuitive judgment of surface shape by subjects (de Vries et al., 1994; Erens et al., 1993; Kappers et al., 1994). However, there seems to be no adequate method for directly obtaining a quantitative surface curvature. Standard psychophysical techniques, such as the yes/no task, the ratio task, and the forced-choice task (Green \& Swets, 1966), have been employed to study the perceptual threshold of curvature (Johnston \& Passmore, 1993). These techniques statistically provide the threshold of curvature, but not the curvature itself.

In this paper, we address the possibility of a quantitative curvature representation that is coded by humans. The primary objectives and motivation of this study were the following three points. First, we attempted to obtain quantitative curvature data and address subjects' sensitivity to surface curvature. All of the experiments utilized virtual quadratic surfaces rendered with visual cues (i.e., texture, shading, perspective, and horizontal disparity). A drawing task (Experiments $1 \mathrm{~A}$ and $1 \mathrm{~B}$ ) and a pointing task (Experiment 2) were used for the reporting of singular directions of curvatures in which curvature was maximum, minimum, and zero. In addition to these experimental paradigms, in Experiment 3, we compared perceptual judgment and motor response for the same stimuli to discuss task specific curvature representation. Second, to address the coding process for surface curvature during the drawing task, we established two different curvature estimation methods from drawing data (Experiments $1 \mathrm{~A}$ and $1 \mathrm{~B}$ ). Both methods estimated the same geometrical properties (maximum and minimum principal curvatures and their directions) through drawing trajectories made by subjects for different directions passing through the tip of a surface. One method (called the differentiation from best-fitted surface, DFF, method) dealt with the data as a set of depths. In this method, we fit a second-order polynomial surface to the drawing trajectories by using a least mean square error criterion, and we calculated the principal curvatures and their directions by differentiation. The other method (called the curvature from curves, $\mathrm{CFC}$, method) dealt with the data as a set of curvatures. This method estimated the same surface properties as the DFF method by using differential geometrical techniques, not a differentiation process. Finally, we discuss the relationship between surface shape and the precision of reporting curvature (Experiments $1 \mathrm{~A}$ and 1B). Previous research suggests that perception of curvature depends on surface shape. It was reported, for example, that subjects showed poor performance on tasks of discriminating Gaussian curvature (de Vries, Kappers, \& Koenderink, 1993; Kappers et al., 1994; Norman \& Lappin, 1992; Phillips \& Todd, 1996; van Damme $\&$ van de Grind, 1993). We discuss the dependency of shape on curvature sensitivity in terms of how consistent are qualitative means and our quantitative experimental paradigm.

\section{EXPERIMENT 1 Reporting Surface Curvature by Using a Drawing Task}

The purpose of Experiment 1 was to develop a method for estimating curvature from drawing trajectories and to investigate quantitative curvature properties from the data of the subjects. The curvature of curves lying on a surface is defined as a function of maximum and minimum principal curvatures and its direction (Euler's formula, Equation 1). For different surface curvatures, we investigated how subjects could report the curvature of curves. Experiment 1 consisted of two parts: a four-direction drawing task (Experiment 1A) and a 16-direction drawing task (Experiment 1B). In both experiments, virtual quadratic surfaces (elliptic and/or hyperbolic) were presented as visual stimuli rendered with various visual cues. Three distinct points appeared on the virtual surface, and the subjects' task was to draw a curved line that connected those points along the surface.

\section{Experiment 1A Four-Direction Drawings for a Random Difference in Principal Curvatures}

\section{Method}

Subjects. The subjects were 2 naive subjects (S.Y. and N.Y.), who were paid for their participation, and 1 of the authors (H.W.). All subjects had normal or corrected-to-normal vision.

Surface definition. We generated nonrotated quadratic surfaces defined by the following equation:

$$
z=\frac{1}{2}\left(\kappa_{\max } x^{2}+\kappa_{\min } y^{2}\right)
$$

Here, $\kappa_{\max }$ and $\kappa_{\min }$ are maximum and minimum principal curvatures. In the screen, $x$ - and $y$-axes agreed with horizontal direction and vertical direction, respectively, and the $z$-axis agreed with viewing direction. Fifteen elliptic surfaces and 15 hyperbolic surfaces were randomly generated. All the parameters of these 30 surfaces are shown in the Table 1.

Visual cue conditions. Two visual cue conditions were employed: a full cue condition and a removed cue condition. In the full cue condition, stimulus of a quadratic surface was simulated by using horizontal disparity, perspective, coarse-grained texture, and lambertian shading. A light source was located at infinity in the direction $(0,0,1)$. In the removed cue condition, the texture cue was not included. Figure 3 shows an example of stimuli. 
Table 1

Surface Parameters in Experiment $1 \mathrm{~A}$

\begin{tabular}{|c|c|c|c|c|c|c|c|c|c|c|}
\hline \multirow[b]{3}{*}{ No. } & \multicolumn{5}{|c|}{ Elliptic Surface } & \multicolumn{5}{|c|}{ Hyperbolic Surface } \\
\hline & \multicolumn{2}{|c|}{$\begin{array}{c}\text { Principal } \\
\text { Curvature }(1 / \mathrm{cm}) \\
\end{array}$} & \multicolumn{3}{|c|}{$\begin{array}{c}\text { Position of } \\
\text { Probe Point }(\mathrm{cm})\end{array}$} & \multicolumn{2}{|c|}{$\begin{array}{c}\text { Principal } \\
\text { Curvature }(1 / \mathrm{cm})\end{array}$} & \multicolumn{3}{|c|}{$\begin{array}{c}\text { Position of } \\
\text { Probe Point }(\mathrm{cm})\end{array}$} \\
\hline & Max. & Min. & Hori. & Diag. & Ver. & Max. & Min. & Hori. & Diag. & Ver. \\
\hline 1 & -0.178 & -0.095 & 4.29 & 5.628 & 6.966 & -0.275 & 0.15 & 1.191 & 7.995 & 14.8 \\
\hline 2 & -0.182 & -0.098 & 4.186 & 5.521 & 6.855 & -0.241 & 0.147 & 2.292 & 8.491 & 14.69 \\
\hline 3 & -0.207 & -0.116 & 3.361 & 4.832 & 6.303 & -0.204 & 0.143 & 3.488 & 9.034 & 14.579 \\
\hline 4 & -0.188 & -0.119 & 3.969 & 5.081 & 6.193 & -0.277 & 0.14 & 1.125 & 7.797 & 14.469 \\
\hline 5 & -0.227 & -0.126 & 2.741 & 4.357 & 5.972 & -0.232 & 0.136 & 2.579 & 8.469 & 14.359 \\
\hline 6 & -0.221 & -0.133 & 2.926 & 4.339 & 5.752 & -0.176 & 0.133 & 4.37 & 9.309 & 14.248 \\
\hline 7 & -0.212 & -0.14 & 3.206 & 4.368 & 5.531 & -0.163 & 0.126 & 4.78 & 9.404 & 14.028 \\
\hline 8 & -0.217 & -0.153 & 3.05 & 4.07 & 5.09 & -0.236 & 0.122 & 2.439 & 8.178 & 13.917 \\
\hline 9 & -0.217 & -0.16 & 3.062 & 3.965 & 4.869 & -0.158 & 0.116 & 4.958 & 9.327 & 13.697 \\
\hline 10 & -0.27 & -0.171 & 1.347 & 2.943 & 4.538 & -0.189 & 0.112 & 3.944 & 8.765 & 13.586 \\
\hline 11 & -0.235 & -0.174 & 2.475 & 3.451 & 4.428 & -0.204 & 0.109 & 3.478 & 8.477 & 13.476 \\
\hline 12 & -0.265 & -0.178 & 1.528 & 2.922 & 4.317 & -0.21 & 0.105 & 3.272 & 8.319 & 13.366 \\
\hline 13 & -0.282 & -0.181 & 0.992 & 2.599 & 4.207 & -0.17 & 0.102 & 4.563 & 8.909 & 13.255 \\
\hline 14 & -0.253 & -0.184 & 1.915 & 3.006 & 4.097 & -0.162 & 0.095 & 4.819 & 8.927 & 13.034 \\
\hline 15 & -0.27 & -0.188 & 1.356 & 2.671 & 3.986 & -0.139 & 0.091 & 5.56 & 9.242 & 12.924 \\
\hline
\end{tabular}

Note-Position of probe point indicates the distance (in centimeters) between display and probe point.

Apparatus. Figure 4 illustrates how the apparatus was arranged. The stimuli were presented on a Silicon Graphics IRIS Crimson/ Reality Engine, and stereo was obtained by using a $120-\mathrm{Hz}$ sequential presentation of left and right images in liquid crystal shutter glasses (CrystalEyes) synchronized to the display monitor, yielding a screen resolution of $491 \times 1,280$. Interocluar separation was adjusted to the value measured for each subject. The subject was asked to hold a stylus and trace a surface in 3-D space within $2 \mathrm{sec}$. An IRED (infrared light-emitting diode) was attached to the tip of the stylus, and the position of the IRED was measured with a 3-D position analysis system (OPTOTRAK, Northern Digital) connected to a computer (J3100, Toshiba). Position data were recorded at $800 \mathrm{~Hz}$.

Procedure. The subject was seated in front of the CRT display at a distance of $60 \mathrm{~cm}$, and the subject's head was fixed with a chinrest. Head height was adjusted such that the viewing direction agreed with the $z$-axis (depth) of the screen and the center of eyes agreed with the center of the display. Under this viewing condition, the CRT display subtended a width of $34.7^{\circ}$ and a height of $24.7^{\circ}$. The stimulus of an elliptic surface subtended approximately a width of $22.4^{\circ}$ and a height of $19.4^{\circ}$, and that of a hyperbolic surface subtended approximately a width of $26.0^{\circ}$ and a height of $19.4^{\circ}$ (degrees of visual arc). For each trial, 1 of 30 surfaces ( 15 elliptic and 15 hyperbolic), one of four drawing directions (vertical, horizontal, two diagonals) and one of two visual cue conditions (full cue or removed cue) were randomly selected.

For the drawing task, the subjects made a drawing motion from a start point through an intermediate point to an end point (all points were displayed on the virtual surface). The intermediate point, which we will call the via point, was used to help further constrain the drawing movement. The start and the end of the movements were prompted by tones generated by the computer. At the first beep, a surface and three points were presented. Points were colored to distinguish each other: Start and end points were green, and the via point was red. The via point was always located at the tip of a surface $[(x, y)=(0.0,0.0)]$. For the start and end points projected on the front parallel plane, four possible locations existed: vertically $[(x, y)=(0.0,9.0),(0.0,-9.0)]$, horizontally $[(9.0,0.0),(-9.0$, $0.0)]$, and diagonally $[(x, y)=(6.36,6.36),(-6.36,-6.36) ;(6.36$, $-6.36),(-6.36,6.36)]$ (units in centimeters). The subject could choose either of the green points as the start point. Since these points were simulated without horizontal disparity, they only informed the subject of direction, not depth. ${ }^{1}$ The second beep cue was generated when the subject placed the stylus on the surface whose $x$ and $y$ po- sitions agreed with the start point and the IRED was detected to be still. Then, the three probe points disappeared from the screen, and the subject began drawing. The subject had to arrive at the end point within 2 sec after the second beep (the third beep informed the subject of this temporal constraint). When the subject finished drawing before the third beep, he/she was asked to keep the stylus at the end point. During each trial, the subject's hand and the IRED (the tip of the stylus) were always visible. One session consisted of 240 trials ( 30 surfaces $\times 2$ visual cue conditions $\times 4$ directions), and all subjects completed three sessions. Before the collection of data began, the subjects practiced several trials until they were comfortable with the response method.

Data analysis. In this section, we introduce two different techniques for estimating the curvature of the perceived surface from the drawing responses at via points. The general procedure consisted of three steps: extracting drawing trajectories around a via point, alignment of the extracted data, and estimating of the principal curvatures and their directions. For the final step, we applied two different methods, DFF and CFC, to the same drawing data extracted around a via point. Each dealt with data from different aspects: as a set of depths and as a set of curvatures, respectively. As mentioned in the introduction, our primary purpose in this study was to find how surface curvature is processed in the human vision system and what kind of surface geometry drawing data represents. If subjects report surface depth every time during a drawing motion, the trajectory data could be considered as a set of depths and the surface curvature should be calculated by differentiation from the "depth map." On the other hand, if subjects report the curvature (radius of curvature) every time during a drawing motion, the trajectory data contain curvature information and the surface curvature can be directly calculated from the drawing data using a combination of differential geometrical techniques.

Extracting data around a via point. The marker positions were sampled for $2 \mathrm{sec}$ at a rate of $800 \mathrm{~Hz}$, resulting in 1,600 sample points for each trajectory. Actual movement time, however, was typically about $1 \mathrm{sec}$, so not all of the movement data were usable. Since the DFF method needed sufficient data points for reliable parameter estimation, we used only raw data that satisfied the following criterion: A trajectory must contain more than 201 sampling points around the maximum or minimum point of depth (at this point, the sign of the rate of depth change reverses) nearest to the via point. When this criterion was violated (the drawing motion was too fast or too slow), a trial was redone. 

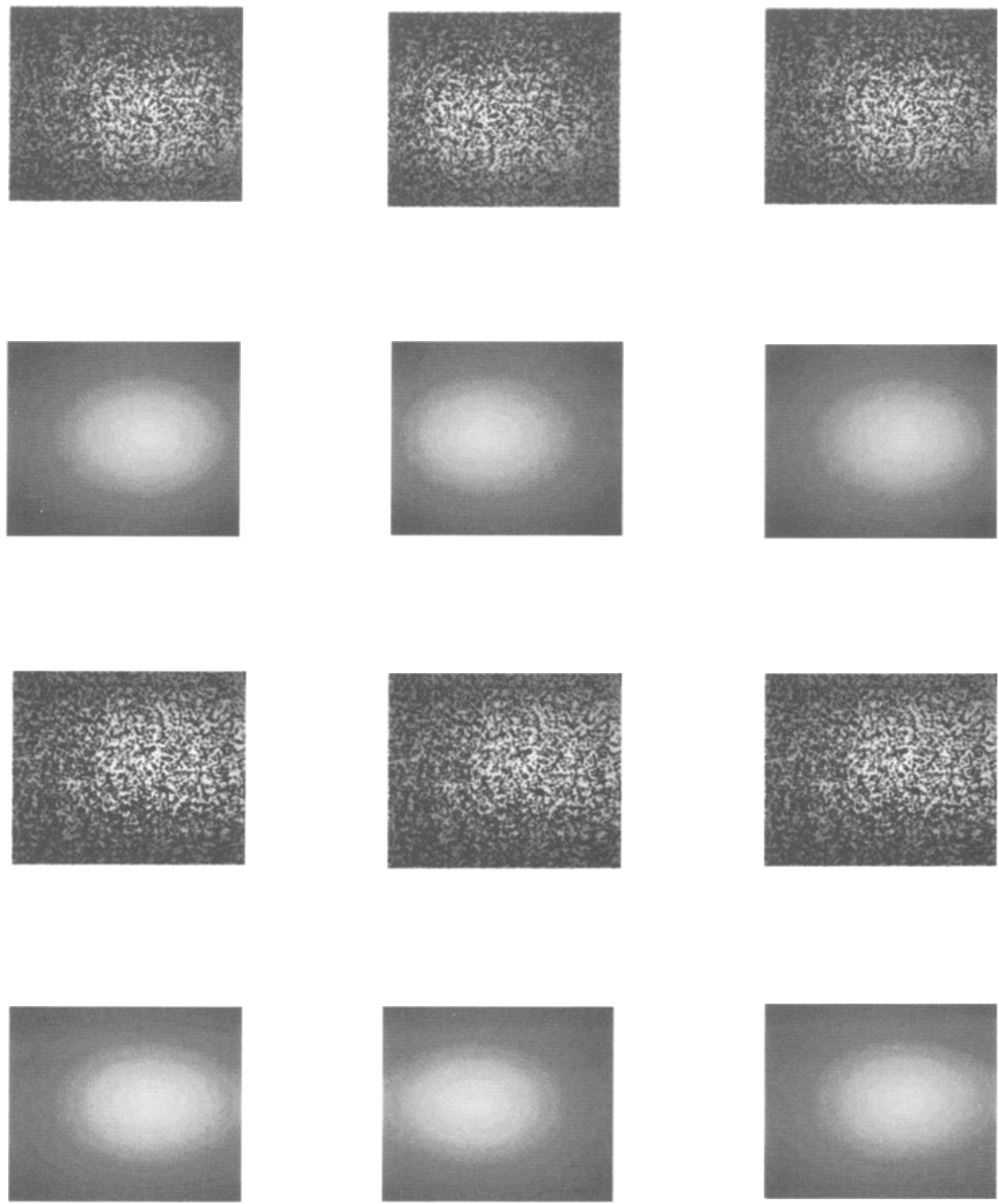

Figure 3. An example of stimuli (left pairs are divergent, right pairs are crossed). In the figure, the upper two rows indicate elliptic surfaces and the lower two rows indicate hyperbolic surfaces. Both of the surfaces were nonrotated and have the same principal curvatures in the upper and lower two rows. Top and second from the bottom stimuli are full cue condition surfaces with cues of texture, horizontal disparity and shading. Second from the top and bottom stimuli are removed cue condition surfaces (i.e., the texture cue was removed from the full cue condition surfaces). In Experiments 1 and 3, we did not control surface edges. In Experiment 2, we placed an aperture in front of the screen and limited the field of view (see text).

Alignment of the extracted data. As mentioned above, we used the maximum or the minimum point nearest to the via point for extracting data. However, the simulated position of the via point did not always equal the position of the maximum or the minimum point of the drawing movement. For the DFF method, we assumed agreement between via point and maximum or minimum point, so all of the extracted data were translated as passing the actual via point.

Two different methods for estimating of the surface curvature from drawing data. Two different methods for estimating curvature were applied to the same data extracted from around a via point and obtained by the above procedure. Here, we introduce the intuitive concepts of these two methods and the mathematical description is presented in the Appendix. The DFF method dealt with data as a set of depths. In this method, we found the best-fitted second-order polynomial surface to the drawing trajectories by a least mean square error criterion. The maximum and the minimum principal curvature and their directions were calculated by the differentiation the bestfitted surface. The CFC method dealt with data as a set of curvatures. In this method, first of all, we fitted circles to each drawing data. The curvature of drawing trajectory were calculated from the radii of the fitted circle, and the slant of the fitted circle in the 3-D space was obtained. From the curvature of drawing trajectory and the angle of the fitted circle, the normal curvature could be calculated (Meusnier's lemma; see Appendix). Using a kind of optimization 


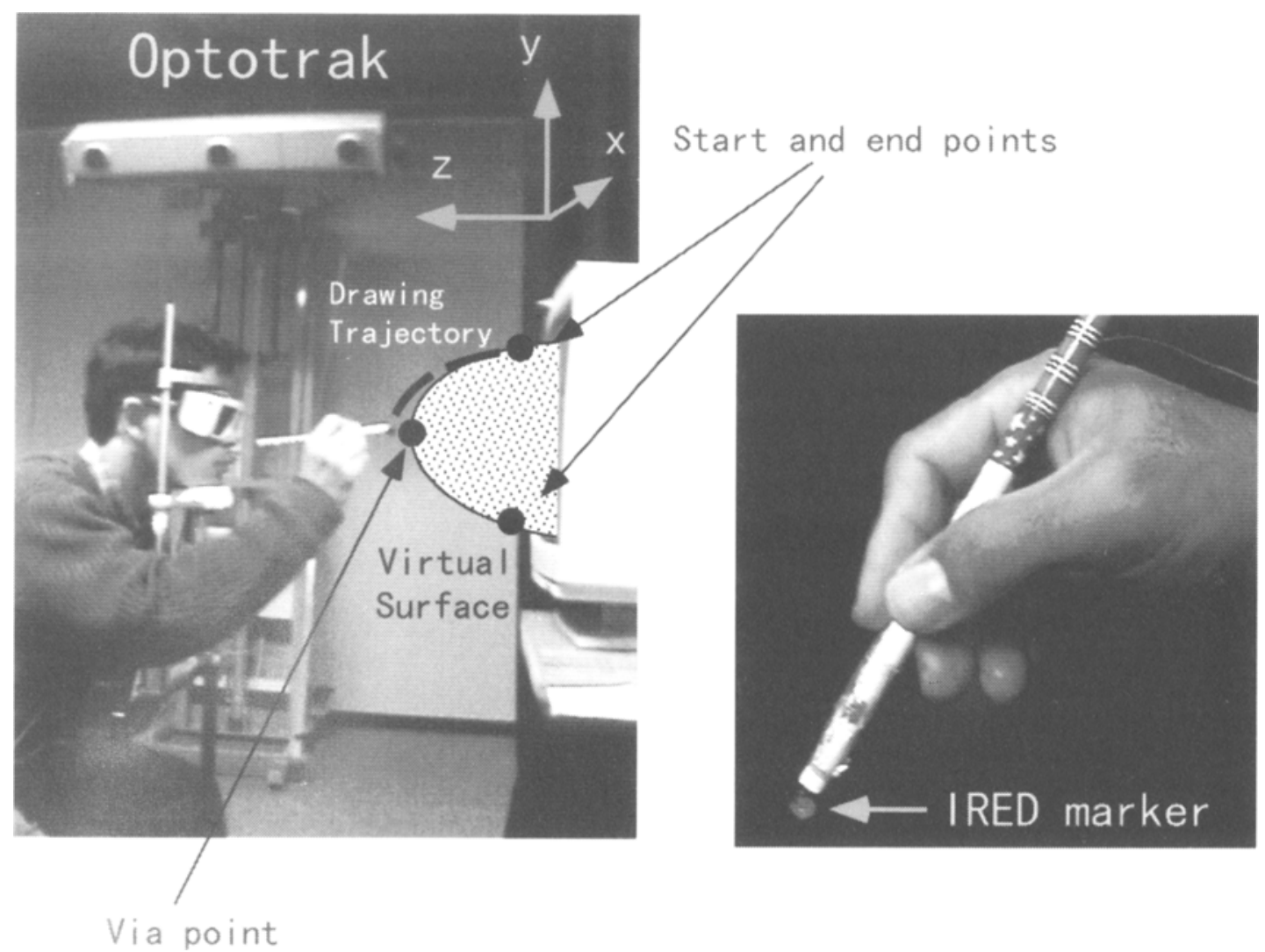

Figure 4. Experimental setup (left), and the wand as held by subjects to trace on the surface (right). In the actual experimental condition, the room light was turned off.

technique, the same surface geometrical properties as in the DFF method were obtained from all drawing data for each surface.

\section{Results and Discussion}

We estimated two sets of maximum and minimum principal curvatures and their directions by using the DFF and CFC methods. The change in curvature of curves between principal curvatures (Euler's formula, Equation A4) could be reconstructed using the three estimated parameters.

To address the effects of surface shape and visual cue conditions, we discuss the following two points using the estimated parameters and the reconstructed Euler's formula: (1) How well do subject data fit the prediction of Euler's formula? (2) How different is simulated and reported curvature for the normal sections?

To discuss the first problem, the coefficient of determination $R^{2}$ of Euler's formula fitted to the raw data was calculated. ${ }^{2}$ Each subject generated 12 blocks of data ( 4 directions $\times 3$ repetitions) for each of 60 surfaces ( 2 visual cue conditions $\times 30$ surfaces $[15$ elliptic +15 hyperbolic]), and we fitted Euler's formula into a linear expression (Equation A6). This fitting process was a multiple regression analysis; thus, we could obtain $60 R^{2}$ s. Let us consider the above issues from the viewpoint of surface umbilicity. When all normal curvatures are equal at a point on a surface, such a point is called an umbilical point. For example, all points on a sphere are umbilical. Figure 5 plots $R^{2}$ as a function of the difference between principal curvatures (Figure 5 shows results for only the full cue condition. Results for the removed cue condition were similar). Points scattered more than $\left(\kappa_{\max }-\kappa_{\min }\right)=$ 0.2 /centimeter were $R^{2}$ obtained with a hyperbolic surface, and the others were $R^{2}$ obtained with an elliptic surface. For a large difference between principal curvatures, $R^{2}$ increases in many cases. In this experiment, however, we did not control the difference between maximum and minimum principal curvatures of the stimuli for the shape, so the effect of shape type was unclear.

To discuss the second point, the mean squared difference between simulated and reported curvatures of normal sections was calculated. Since curvatures of curves drawn with the probe were not constant, the amount of difference was normalized by using the variance of the simulated curvature. Figure 6 plots the normalized squared difference as a function of the difference between prin- 


\section{Full cue condition}
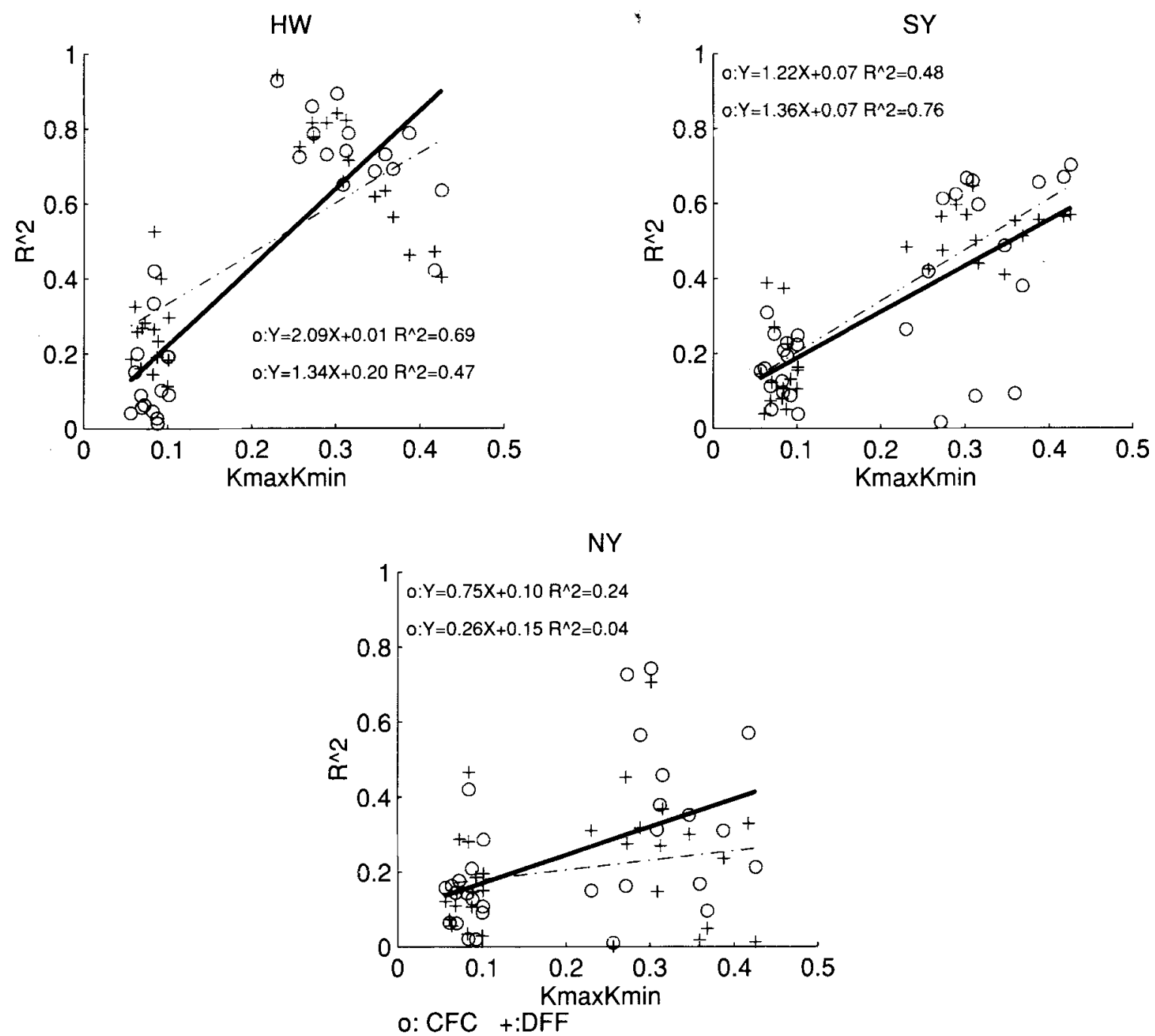

Figure 5. The coefficient of determination $R^{2}$ plotted as a function of the difference between principal curvatures (only the full cue condition). Values of $R^{2}$ obtained by the CFC method are indicated by " $O$," and the solid line shows the linear regression line obtained from these values. Values of $R^{2}$ obtained by the DFF method are indicated by "+," and the dash-dotted line shows the linear regression line obtained from these values.

cipal curvatures. As the difference between principal curvatures increased, the normalized squared difference decreased in all cases.

Taken together, the overall pattern of results suggest that the subjects could have been sensitive to the change in curvature of curves, as would be predicted by Euler's formula when the difference between principal curvatures was sufficient (i.e., the surface was not umbilical). However, a statistical test for the estimated parameters $A, B$, and $C$ in Equation A6 (null hypothesis $H_{0}$ was $A, B, C=$ 0 ) showed that only parameter $A$ was significant for the elliptic surface, and few parameters were significant for the hyperbolic surface. In our experiment, the maximum principal directions always agreed with horizontal axis
$\left(\theta_{M}=0\right)$ and, thus, ideally $C=0$ by Equation A7. Such findings suggest that these parameters hardly contributed to predicting the data. Three possible reasons could be considered: (1) The subjects were not sensitive to probe curves with small curvatures. (2) Although the subjects attempted to correctly report a small curvature, the estimated parameters were not significant. (3) The amount of data was insufficient for reliably estimating three parameters with data from four directions. Furthermore, the effect of shape is still unclear because the principal curvatures were not strictly controlled for the elliptic and hyperbolic surfaces. To clarify these questions and to look at the sensitivity of the subject to a change in the curvature of curves, we conducted Experiment 1B. 
HW

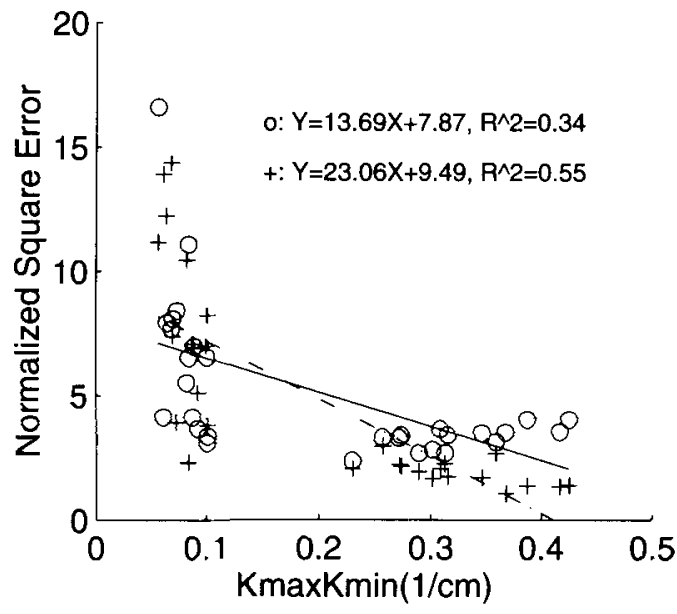

SY

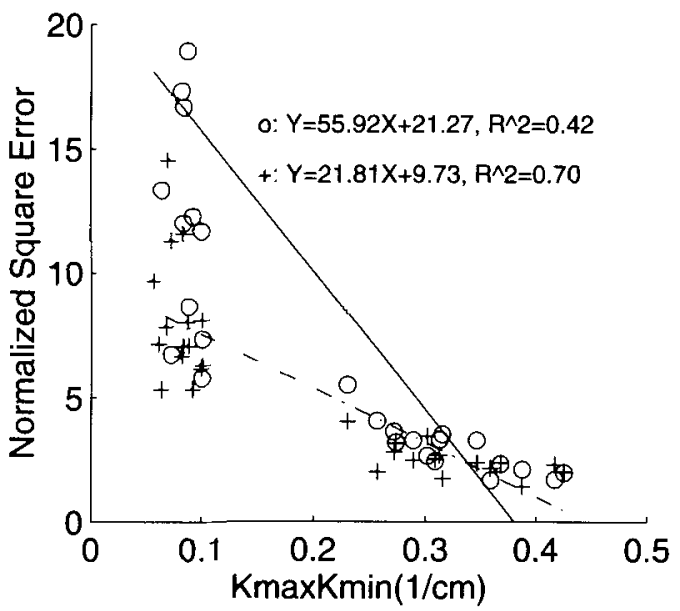

NY

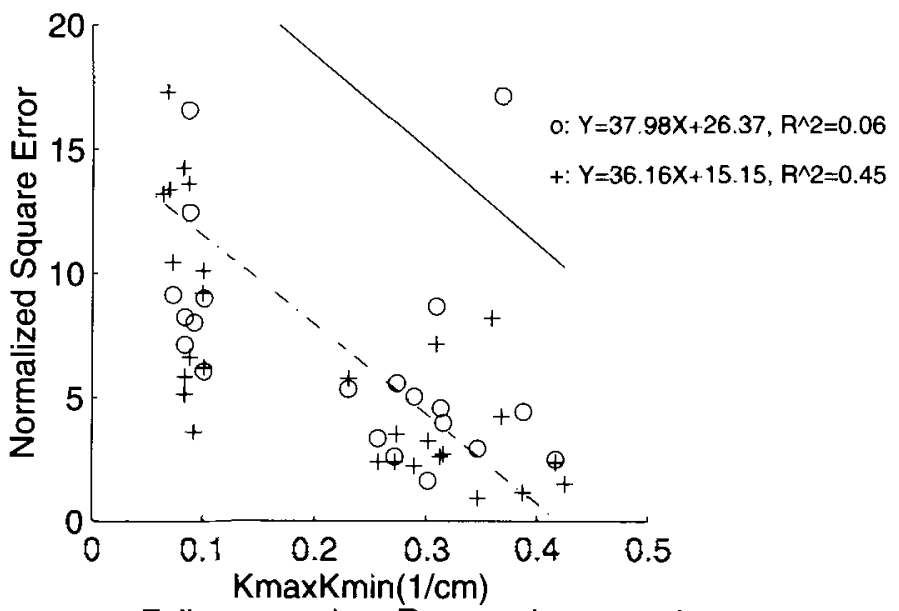

o: Full cue cond. +: Removed cue cond.

Figure 6. Normalized error between simulated and reported curvature of a normal section plotted versus the difference between maximum and minimum curvature. "O" and solid line indicate the normalized error and linear regression under the full cue condition. "+" and dash-dotted line indicate the normalized error and linear regression under the removed cue condition. 
Table 2

Principal Curvatures (1/Centimeter) of the Surfaces Simulated in Experiment 1B

\begin{tabular}{lcc}
\hline & \multicolumn{2}{c}{ Principal Curvature } \\
\cline { 2 - 3 } Surface & Maximum & Minimum \\
\hline Elliptic & 0.3 & 0.05 \\
Hyperbolic & 0.15 & -0.10 \\
\hline
\end{tabular}

\section{Experiment 1B}

Sixteen-Direction Drawings for

\section{a Common Difference in Principal Curvatures}

The results of the Experiment $1 \mathrm{~A}$ suggested that the $R^{2}$ of Euler's formula used for fitting and the normalized difference from a simulated value appeared to be explained by the difference between principal curvatures (surface umbilicity), not by the surface shape (as indicated by the sign of Gaussian curvature). However, such a conclusion would be premature since the relation between surface shape and the difference of principal curvatures was not strictly controlled. In Experiment 1B, we held constant the difference between principal curvatures for both an elliptic and an hyperbolic surface. The same drawing task was used, with an increased number of directions on the surface.

\section{Method}

Subjects. The subjects were 3 naive subjects (C.Y., N.A., and T.T.), who were paid for their participation, and 1 of the authors (H.W.). All subjects had normal or corrected-to-normal vision.

Apparatus. The same equipment used in Experiment $1 \mathrm{~A}$ was used in Experiment 1B.

Stimuli and Procedure. We generated two quadratic surfaces, one elliptic and one hyperbolic, by using Equation 2. Table 2 shows the maximum and minimum principal curvatures employed in this experiment. It should be noted that we selected the common difference principal curvatures for the elliptic and hyperbolic surfaces in this experiment (i.e., 0.25 /centimeter). The stimulus of an elliptic surface approximately subtended a width of $16.1^{\circ}$ and a height of $22.6^{\circ}$ of visual arc, and that of a hyperbolic surface subtended a width of $18.9^{\circ}$ and a height of $24.5^{\circ}$ of visual arc. The same visual cue conditions were used as in the previous experiment: a full cue condition and a removed cue condition. For each trial, the surface was randomly rotated along the view direction.

The task was identical to that in Experiment 1A: The subjects made drawing movements on the surface from the start point to end point through the via point by following the beep cues. The design followed that of Experiment $1 \mathrm{~A}$, but two points were modified: the number of directions and the visualization of probe points during drawing. The subjects made 16 separate drawing motions between principal directions, and the three probe points were kept visible on the surface during drawing to ensure that the subjects understood the directions. One session consisted of 64 trials $(2$ surfaces $\times 2$ visual cue conditions $\times 16$ directions), and all of the subjects completed six sessions

\section{Results and Discussion}

The same criterion as in Experiment 1A was used for extracting data from around a via point for each trajectory generated by the subjects. Extracted data were aligned at a via point, and the DFF and CFC methods were used to estimate the principal curvatures and principal directions. Figure 7 summarizes the results of Subject T.T. The left collumn of Figure 7 indicates the simulated curvature of curves (cross marks), reported curvature of normal sections (circles), and the reconstructed curvature of curves estimated with the DFF method (dash-dotted line) and the CFC method (solid line). Note that, for the elliptic surface, the curvature is always positive; for the hyperbolic surface, the curvature changes signs with maximum and minimum principal curvatures at $0^{\circ}$ and $90^{\circ}$, respectively. The right column of Figure 7 indicates the standard deviation of repetitions in each direction (solid lines) and the mean squared difference for simulated and responded curvature of curves in each direction (dash-dotted lines). Table 3 shows results of statistical significance with respect to parameter estimation.

The results can be summarized as follows: (1) For the full cue condition and the hyperbolic surface, all subjects overestimated the minimum principal curvature. Thus, they perceived and/or reported a more curved surface than the one presented (left column of Figure 7). (2) For the removed cue condition and both surfaces, the subjects had trouble reporting the change in curvature. Data suggest that they sometimes misperceived even the global surface shape when the texture cue was removed. For example, Subject T.T.'s reconstructed curves for a saddle shape indicate that she always reported the surface as being convex elliptic (estimated maximum and minimum principal curvatures were always positive). To the contrary, Subject N.A. always reported the surface as concave elliptic (estimated maximum and minimum principal curvatures were always negative; left column of Figure 7). (3) Generally, both indices of variability shown in the right column of Figure 7 match each other. This suggests that the average of data reported for a normal section and a given direction matches the average of that for the simulated one. (4) The coefficients of determination $\left(R^{2}\right)$ of Euler's formula used for fitting (Table 3) were higher for the hyperbolic surface than for the elliptic surface $[F(1,3)=29.971, p<.05]$ and were also higher for the full cue condition than for the removed cue condition $[F(1,3)=10.145, p<.05]$. (5) A signed rank sum test revealed that the $R^{2}$ of Euler's formula used for fitting obtained with the CFC method was significantly higher than that obtained with the DFF method $(p<.05)$. This suggests that the subjects' drawing performance could be predicted better when data were treated as a set of curvatures rather than as a set of depths. (6) The multiple regression analysis included calculation of $t$ statistics and the corresponding significance probabilities to test whether each parameter was significantly different from zero. These results revealed that, in general, parameters $A$ and $B$ were significantly estimated for the elliptic surface, but only $B$ was for the hyperbolic surface (Table 3 ).

Previous researchers have pointed out the effect of surface shape on surface perception (de Vries, 1993; Kappers et al., 1994; Phillips \& Todd, 1996) by reporting the 
Subject: TT

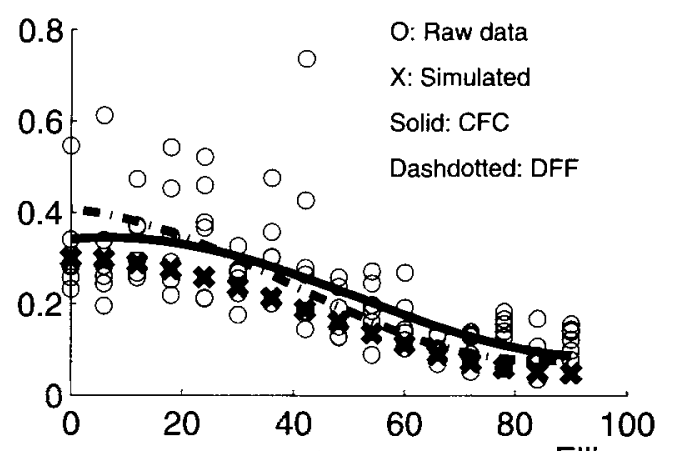

Ellipse, Full cue

Ellipse, Removed cue

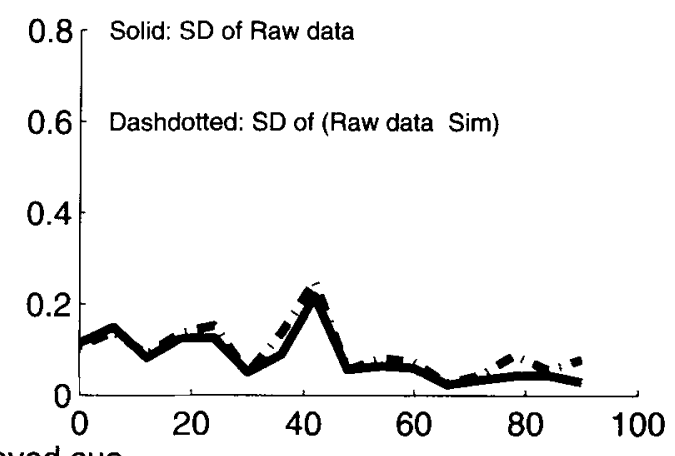

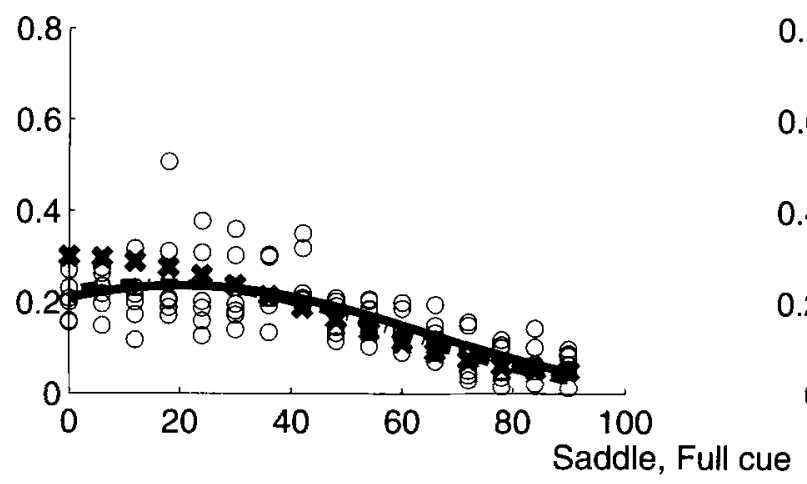
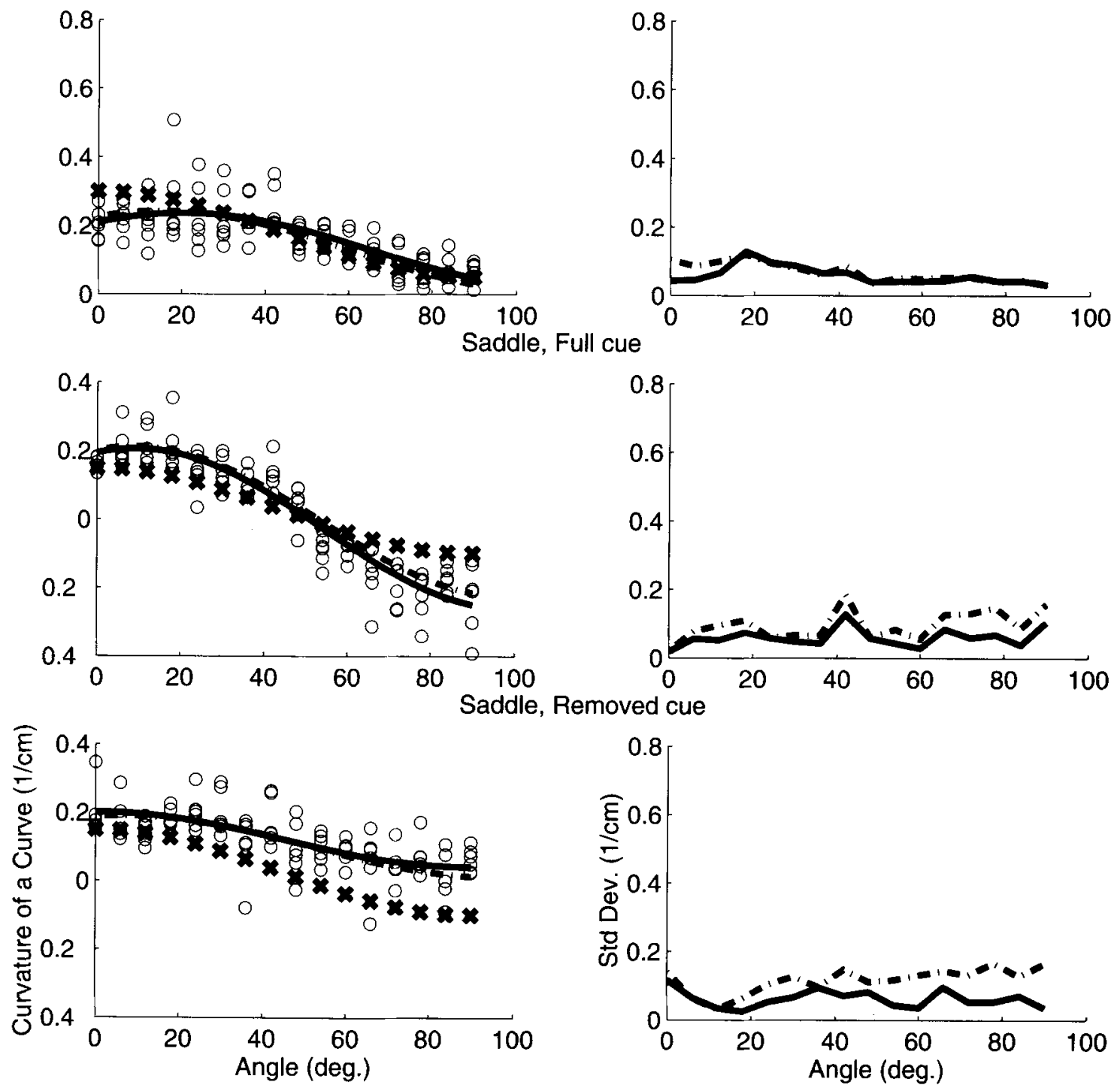

Figure 7. Results from Experiment 1B showing raw data, simulated and reconstructed curvature of curves, and errors for Subject T.T. (left column). The standard deviation of repetition and the mean squared error are also indicated (right column). 
Table 3

Summary of Multiple Regression Analysis

\begin{tabular}{|c|c|c|c|c|c|c|c|c|c|c|c|c|c|c|c|c|}
\hline \multirow[b]{3}{*}{ Subject } & \multicolumn{8}{|c|}{ Full Cue Condition } & \multicolumn{8}{|c|}{ Removed Cue Condition } \\
\hline & \multicolumn{4}{|c|}{ CFC } & \multicolumn{4}{|c|}{ DFF } & \multicolumn{4}{|c|}{$\mathrm{CFC}$} & \multicolumn{4}{|c|}{$\mathrm{DFF}$} \\
\hline & $R^{2}$ & $A$ & $B$ & $C$ & $R^{2}$ & $A$ & $B$ & $C$ & $R^{2}$ & $A$ & $B$ & $C$ & $R^{2}$ & $A$ & $B$ & $C$ \\
\hline \multicolumn{17}{|c|}{ Elliptic } \\
\hline H.W. & .59 & ** & * & - & .56 & $* *$ & $* *$ & - & .55 & $*$ & $*$ & - & .38 & $*$ & * & - \\
\hline C.Y. & .37 & $*$ & * & - & .27 & $*$ & $*$ & - & .42 & $*$ & * & - & .36 & $*$ & $*$ & - \\
\hline N.A. & .44 & $* *$ & * & - & .0 & $\uparrow$ & $\dagger$ & - & .56 & $* *$ & * & - & .0 & - & $\dagger$ & - \\
\hline T.T. & .49 & $*$ & $*$ & - & .42 & $*$ & * & - & .52 & $*$ & $*$ & $\dagger$ & .42 & $*$ & * & - \\
\hline \multicolumn{17}{|c|}{ Hyperbolic } \\
\hline H.W. & .92 & - & $* * *$ & + & .91 & - & $\neq * *$ & - & .76 & + & $* *$ & - & .72 & $\dagger$ & $* *$ & - \\
\hline C.Y. & .65 & - & $* *$ & - & .56 & - & $*$ & - & .57 & - & $*$ & - & .56 & - & $*$ & - \\
\hline N.A. & .83 & $\dagger$ & ** & + & .75 & + & $* *$ & - & .34 & $*$ & $*$ & - & 16 & + & + & - \\
\hline T.T. & .84 & - & $* *$ & + & .49 & - & $* *$ & - & .43 & $*$ & $*$ & - & .41 & * & * & - \\
\hline
\end{tabular}

poor performance of subjects for hyperbolic surfaces. A plausible and ecological explanation might be that a hyperbolic surface lacks a kind of "familiarity" in daily life (Kappers et al., 1994). We examined our data to see whether they provided a result consistent with this finding. A $t$ test for the elliptic and hyperbolic surfaces showed no significant difference in the averaged standard deviation of six repetitions in each direction (for elliptic and hyperbolic, average $=0.08 /$ centimeter and $0.09 /$ centimeter, respectively), but it showed a significant difference between the averaged squared difference and the simulated value for elliptic and hyperbolic surfaces [average $=0.11$ / centimeter, and $0.15 /$ centimeter, respectively; $t(254)=$ $4.52, p<.001]$. Although the variance of repetition within subjects was constant regardless of the surface shape, the subjects could better report surface curvature for the elliptic surface than for the hyperbolic surface. On the other hand, the $R^{2}$ of Euler's formula used for fitting (Table 3) was higher for the hyperbolic surface than for the elliptic surface (the averaged values across subjects and visual cue conditions $=.49$ and .67 , for elliptic and hyperbolic, respectively).

A similar trend is also suggested for the estimated principal curvatures across the surface type. Figure 8 shows the estimated principal curvatures for all subjects and a linear regression fit to these data. As indicated, the estimated principal curvatures of an elliptic surface seem to match those of the simulated surface: Consistently, slopes are around 1.0, and intercepts are around 0.0 . The differences in principal curvatures for a hyperbolic surface, on the other hand, were always overestimated; under the full cue condition, the subjects overestimated the difference by about twice as much. The amount of variability accounted for by the linear regression, however, was greater for the hyperbolic surface than for the elliptic surface. These results suggest a tradeoff between accuracy and variability. The addition of visual information had a substantial effect for the hyperbolic surface, but not for the elliptic surface.

In addition to these findings, it is interesting to note the performance around zero curvature of curves lying on the surfaces. A hyperbolic or parabolic surface always has zero curvature lines passing at the tip of the surface (Figure 2), and this can be shown by Euler's formula (Equation 1). When $\kappa_{S}^{i}$ is 0 , the following equation is derived:

$$
\cos ^{2} \alpha^{i}=-\frac{\kappa_{\min }}{\kappa_{\max }-\kappa_{\min }} .
$$

When $\kappa_{\max }>0$ and $\kappa_{\min } \leq 0$, the solution of Equation 3 is

$$
\alpha^{i}=\arccos \left( \pm \sqrt{-\frac{\kappa_{\min }}{\kappa_{\max }-\kappa_{\min }}}\right) .
$$

Thus, hyperbolic $\left(\kappa_{\min }<0\right)$ and parabolic $\left(\kappa_{\min }=0\right)$ surfaces contain a zero curvature line in the direction given by Equation 4.

The naive subjects were not told about the existence of the zero curvature line on the hyperbolic surface before the experiment, and their introspections seemed to show that they were not aware of them. In data for the hyperbolic surface, the curvatures of trajectories had unstable signs around the asymptotic direction (direction of the zero curvature line; $\theta=50.8^{\circ}$ ). It is unclear which factor was dominant, perceptual sensitivity or motor error for the small curvature? How sensitive is human perception to the zero curvature lying on a hyperbolic surface? This problem relates to curvature coding: Is the absolute value of the curvature coded, or is the relative change of the curvature coded? To address this issue, in Experiment 2, we focused on the sensitivity of each subject to the directions that corresponded to the principal and asymptotic directions on the hyperbolic surface.

\section{EXPERIMENT 2 \\ Reporting the Principal and Asymptotic Directions by Using a Pointing Task}

In Experiment 2, subjects reported the principal directions and asymptotic (zero curvature line) directions on the virtual hyperbolic surface by using a pointing task. As we have pointed out, in Experiment 1, the subjects' sensitivity to surface curvature seemed to depend on the 

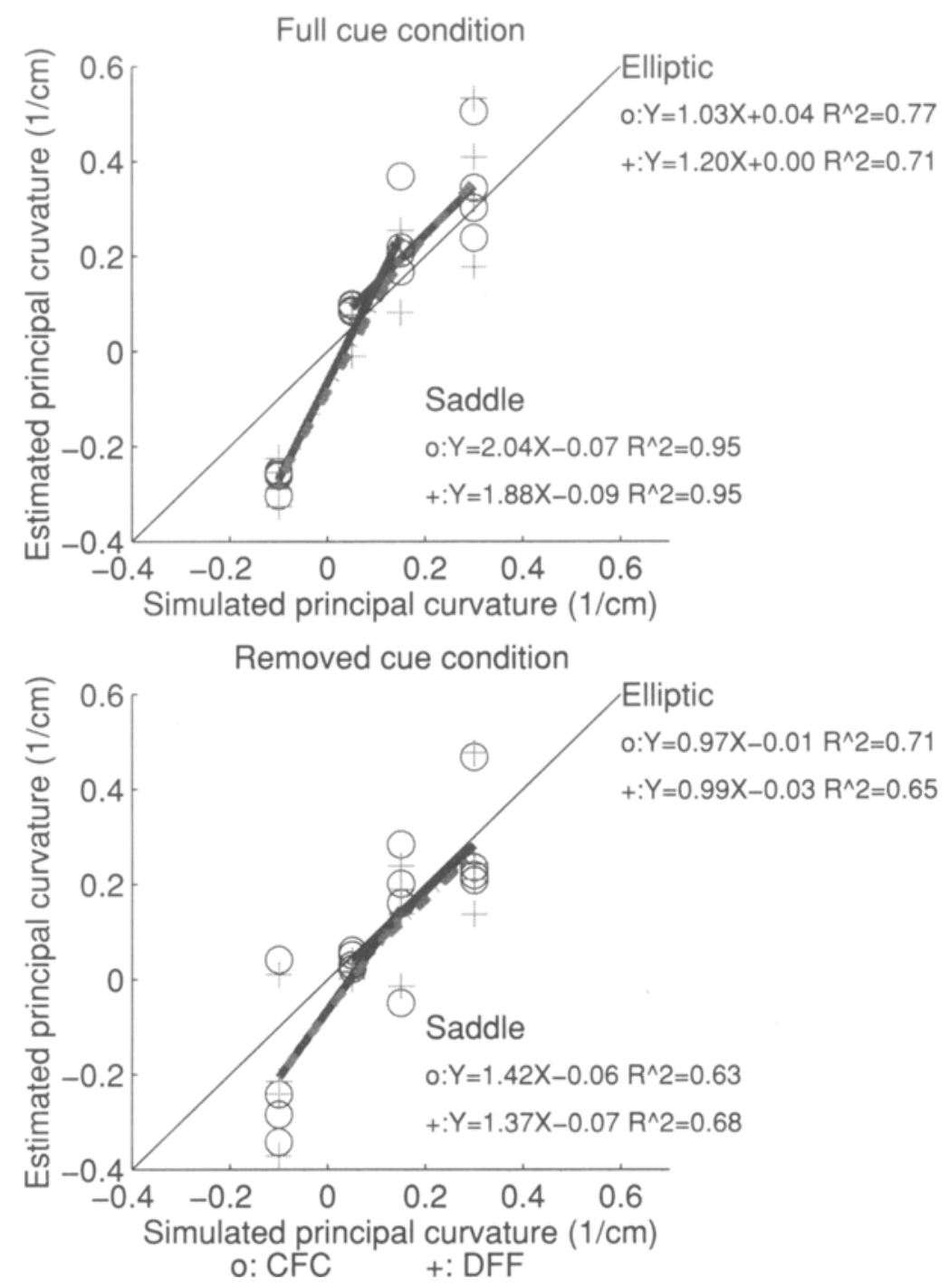

Figure 8. Estimated principal curvatures for all subjects, along with linear regressions. The two lines (solid and broken) to the left in each panel indicate the estimated maximum and minimum principal curvatures for the hyperbolic surface. The two lines to the right indicate the estimated maximum and minimum principal curvatures for the elliptic surface.

value of curvature. However, it was still unclear whether the coded curvature was an absolute or a relative one. The principal curvatures and the zero curvature are singular points where the curvature of a curve changes, and they correspond to the extreme points of change and zero change, respectively, for the curvature of curves. If subjects cannot perceive the zero curvature, they are partially insensitive to the absolute value of the curvature of curves. Otherwise, they are able to perceive a reliable representation for the value of curvature. To clarify this issue, we asked subjects to indicate the principal directions and the directions of zero curvature on the hyperbolic surface.

\section{Method}

Subjects. The subjects were 3 naive subjects (K.T., M.Y., and H.M.), who were paid for their participation, and 1 of the authors (H.W.). All subjects had normal or corrected-to-normal vision.
Apparatus and Stimuli. Except for the presence of a response device and an aperture, the same equipment used in Experiment 1 was used in Experiment 2. The subjects responded to the principal and asymptotic directions by using a 3-D digitizing wand (OPTOTRAK 25-marker digitizing probe, Design 2, Northern Digital). The wand was instrumented with 25 infrared light-emitting diodes, whose positions were measured by an OPTOTRAK, and the absolute orientation of the wand was calculated by using a technique based on quarternions (Horn, 1987; Pollick et al., 1996). In a study on structure from motion, Pollick and colleagues used this device for reporting the axis of rotation (Pollick, Nishida, Koike, \& Kawato, 1994), and they also used it in a study on shape from shading to report the local surface orientation (Pollick et al., 1996). The precision and reliability of this method were confirmed, and it was considered a good method for reporting surface geometry.

An aperture was placed in front of the screen, limiting the field of view to $6.4^{\circ} \times 18.9^{\circ}$. As such, the edges of the surface displayed were not visible. Because the surface was viewed through a rectangular aperture and the surface was rotated randomly around the $z$ - 


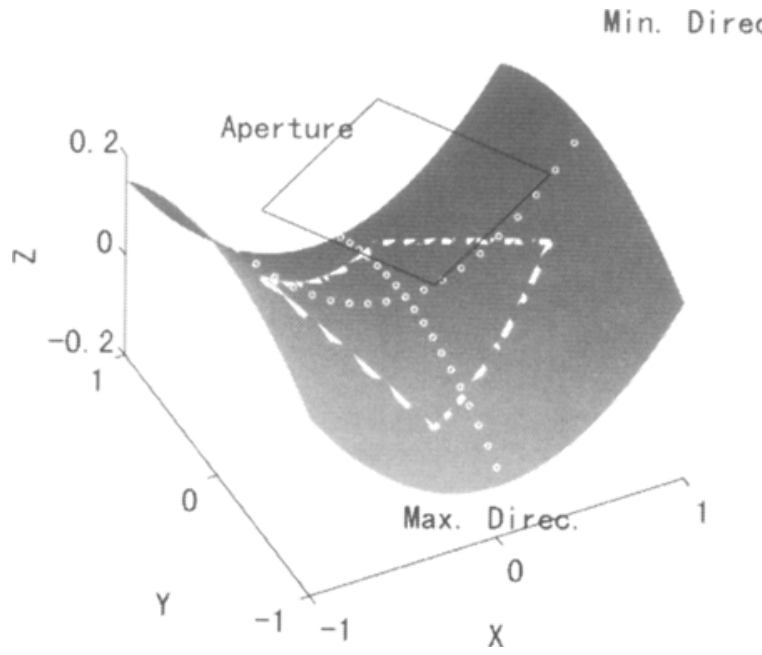

Figure 9. Example of the surface region visible through the aperture for Experiment 2. We placed a rectangular aperture in front of the surface so that the subjects could see only a limited surface region (within the dotted line area). For each trial, furthermore, the surface was randomly rotated around the viewing direction ( $z$-axis), so the principal directions (open circle lines) did not agree with the nearest and farthest point at the edge.

axis (viewing direction) for each trial, depth at aperture did not indicate the principal directions. Thus, a strategy based solely on maximum and minimum depth in the region of the aperture would not reliably indicate the direction of the principal curvatures (Figure 9).

We generated 10 hyperbolic surfaces by using Equation 2 . Table 4 shows the maximum and minimum principal curvatures employed in this experiment. Two visual cue conditions, the same as in the Experiment 1, were employed.

Procedure. The subjects' task was to orient the digitizing wand in a direction parallel to the perceived maximum and minimum principal curvatures and the zero curvature line at the tip of the hyperbolic surface. We defined positive curvature to be convex and negative curvature to be concave, relative to the subjects. A computer generated "beep" cues for each trial, informing the subjects about which direction should be reported (maximum, minimum, or zero curvature line). Before the beginning of the experiment, the subjects were given an intuitive explanation of the task as follows:

\footnotetext{
Your task is to find the most curved direction and a straight line direction of the object, then place the long axis of the 3-D digitizing wand in that direction. You report the most convex curved direction when the beep sounds once, and you report the most concave curved direction when the beep sounds twice. Furthermore, there are two straight lines passing at the tip of the surface. You can report either one of the directions when the beep sounds three times.
}

Except for H.W., the subjects were not told that the two principal directions were always orthogonal. They practiced several trials until they were comfortable with the response method. In each session, the subjects performed 60 trials ( 10 surfaces $\times 2$ visual cue conditions $\times 3$ directions), and all subjects completed six sessions. Stimuli and directions were presented in random order.

\section{Results and Discussion}

We defined two errors: constant error is the absolute angular difference between the responded and the simulated direction, and variable error is the standard deviation of repetition. For each of the three directions and the two visual cue conditions, constant errors were averaged across individual sessions to obtain an average error for each surface and were averaged across surfaces to obtain an overall average. Figure 10 shows the averaged constant error for the 4 subjects in each direction under the two visual cue conditions.

An analysis of variance was conducted using the factors of direction and visual cue. The results revealed a significant main effect of visual cue condition $[F(1,3)=24.38$, $p<.05]$ and a significant interaction $[F(2,6)=44.39$, $p<.001]$. Inspection of Figure 10 shows that, under the removed cue condition, the subjects' performance did not change much across the probe directions (maximum, minimum, and asymptotic directions); however, for the full cue condition, error for the asymptotic direction was five to six times greater than error for the principal directions. Tukey's honestly significant difference (HSD) multiple comparisons were carried out among the probe directions. Results showed that, for the full visual cue condition, there were significant differences between the asymptotic direction and the principal directions $(p<.01)$.

Using a similar experimental paradigm, de Vries (1993) studied perception of the principal directions, but with two major methodological differences: response manner and the global shape of stimulus. First, our subjects reported the maximum and minimum principal directions separately, whereas de Vries subjects matched the cross marker, which was superimposed on the surface, to the principal direction at the probe points. Thus, the subjects were told in advance that the principal curvatures are orthogonal, and they simultaneously reported both of them. Data around umbilical areas $(|S|>0.85)$ and with a low value of curvedness $(C<0.3$ /centimeter) were discarded (de Vries, 1993). The curvedness at probe points simulated in our experiment were $0.197 /$ centimeter at most and 0.038 /centimeter at least. Although we simulated surfaces with low values of curvedness that satisfied de Vries's criterion for omission, our subjects performed as well as or better than his. The best and worst constant errors were $3.27^{\circ}$ and $10.05^{\circ}$, and those of de Vries were $4^{\circ}$ and $12^{\circ}$.

Second, de Vries (1993) presented rather globally complex surfaces (third-order asymmetric surface) using only horizontal disparity as a visual cue. More consistent

Table 4

Principal Curvatures (1/Centimeter) of the Surfaces Simulated in Experiment 2

\begin{tabular}{ccc}
\hline & \multicolumn{2}{c}{ Principal Curvature } \\
\cline { 2 - 3 } Surface & Maximum & Minimum \\
\hline 1 & 0.115 & -0.254 \\
2 & 0.130 & -0.160 \\
3 & 0.099 & -0.162 \\
4 & 0.053 & -0.158 \\
5 & 0.010 & -0.159 \\
6 & 0.016 & -0.147 \\
7 & 0.026 & -0.109 \\
8 & 0.016 & -0.098 \\
9 & 0.003 & -0.088 \\
10 & 0.039 & -0.037 \\
\hline
\end{tabular}




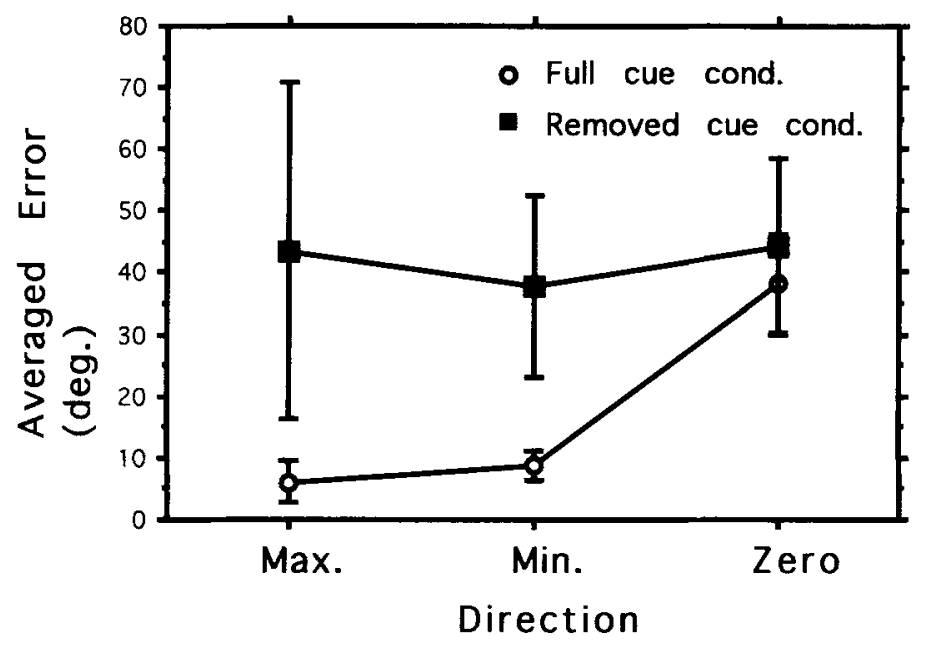

Figure 10. Averaged constant and variable errors plotted under the two visual cue conditions.

and multiple visual cues may increase accuracy of surface representation. Our subjects did well in reporting the principal directions, and this could have been the result of a common visual integration processing, such as accumulation, cooperation, and disambiguation (Bülthoff \& Mallot, 1988). Regarding the global shape, de Vries used an asymmetric surface, but we used a symmetric one. He suggested that it is easy to find the principal directions of a quadratic surface because of its symmetry, but there seems to be room for argument on this point. In our experiment, the visual field was limited by a rectangle aperture, and the surfaces were randomly rotated along the viewing direction. This operation made the edge of a surface random, and the effect of a projected shape on the monitor screen and the effect of depth at the edges were eliminated. Therefore, depending on its quantitative coding, it seems reasonable to suppose that our subjects could implicitly interpret the directions of principal curvature.

Our results suggest that the existence of a texture cue influenced performance for the principal directions. This result is reasonable considering that most studies on perception of shape from shading conclude that shading is a weak cue, is unable to reliably convey local shape, and is easily dominated by other sources of visual information (Bülthoff \& Mallot, 1988; Erens et al., 1993; Mingolla \& Todd, 1986; Pollick et al., 1996; Todd \& Reichel, 1989). In our experiment, the viewing angle for some surfaces was too small to sufficiently change the intensity of a surface. In such a case, subjects might not perform "intensitybased stereo" (Bülthoff \& Mallot, 1988). Mingolla and Todd (1986) presented cylindrical surfaces by only using a shading cue and had their subjects estimate the curvatures. The results showed a remarkable underestimation of surface curvature. Erens et al. (1993) studied the perception of shape from shading and found that subjects sometimes misperceived even concave and convex shapes from shaded images of a Gaussian hill. On debriefing, our subjects reported that they could not clearly perceive the removed cue surface shape. Their performance, however, was not always random. The subjects' raw data indicate that they sometimes made a $90^{\circ}$ error for the principal directions. The reversed perception of concaveness and convexness helps account for this result. Assuming this possibility, we recalculated constant error in the following manner: We took the direction of response and found the absolute value of its angular difference with both the maximum and the minimum direction of curvature. These two absolute values were then compared, and the smaller of the two was defined to be the constant error.

Table 5 compares the original constant error and the recalculated constant error. For the latter case, a decrease of about less than $50 \%$ is indicated for the removed cue condition. It can be seen that, for the full cue condition, there is little change when recalculating the error, but, for the removed cue condition, error is substantially reduced.

Our results showed an opposite data trend for the asymptotic direction. Regardless of visual cue conditions, performance for the zero curvature line was poor. Both constant error and variable error were much larger than those for principal curvatures, and existence of the texture cue did not have a significant effect $(t=-2.438, p=$ $.051)$. It seemed to be difficult for the subjects to perceive the zero curvature line on the hyperbolic surface even

Table 5

Comparison of Original Error and Recalculated Error

\begin{tabular}{|c|c|c|}
\hline Direction of Principal Curvature & Full Cue & Removed Cue \\
\hline \multicolumn{3}{|c|}{ Original Error } \\
\hline Maximum & $10.06^{\circ}$ & $57.78^{\circ}$ \\
\hline Minimum & $9.70^{\circ}$ & $44.92^{\circ}$ \\
\hline \multicolumn{3}{|c|}{ Recalculated Error } \\
\hline Maximum & $7.25^{\circ}$ & $16.98^{\circ}$ \\
\hline Minimum & $7.29^{\circ}$ & $16.72^{\circ}$ \\
\hline
\end{tabular}




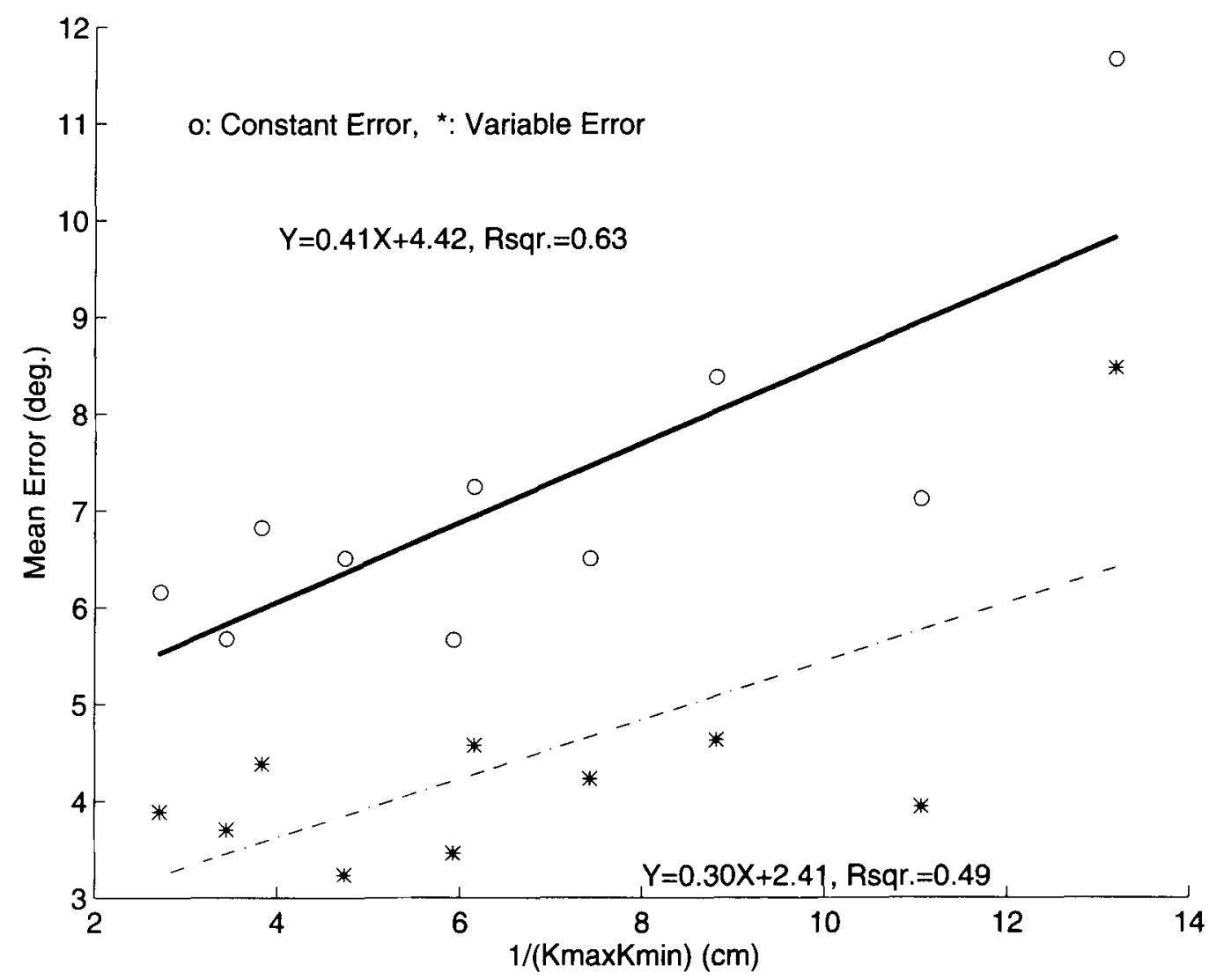

Figure 11. Constant and variable error plotted versus the inverse of the difference of principal curvatures. Also shown are linear regression fits to this data.

though their reporting of principal curvatures was accurate and stable. Even when the texture cue was removed, the subjects seemed to behave as if they knew the direction that corresponded to the maximum change of the curvature of curves. Let us consider the phenomena corresponding to a changing in the curvature.

The curvature $\kappa$ of curves lying on the surface is described as a function of the principal curvatures and the direction $\alpha$ from $\kappa_{\max }$ (Euler's formula, Equation 1). The next equation can be derived from the derivative of Equation 1:

$$
\frac{\delta \kappa}{\delta \alpha}=\left(\kappa_{\max }-\kappa_{\min }\right) \sin 2 \alpha .
$$

When $\alpha=0$ and $\pi / 2, \delta \kappa / \delta \alpha$ becomes zero, and Equation 1 provides the extreme values that correspond to the principal curvatures. Assuming that subjects use relative change of line curvature to detect the principal curvature, they find the principal directions when Equation 5 is zero and has a tolerance of $\pm \varepsilon$. Equation 5 can be approximated by the straight line whose slope is $\left(\kappa_{\max }-\right.$ $\left.\kappa_{\min }\right)$ at $\alpha=0$ and $\pi / 2$. The $\alpha$ that gives $0 \pm \varepsilon$ in Equation 5 is denoted by the following:

$$
\alpha= \pm \frac{\varepsilon}{2\left(\kappa_{\max }-\kappa_{\min }\right)} .
$$

Thus, the range of reported principal direction (constant error) can be defined by this function as the inverse of the difference between principal curvatures. The averaged constant and variable error for the 4 subjects under the full cue condition plotted against the inverse of the difference between principal curvatures along with corresponding values of the linear regression are shown in Figure 11. Positive linear relationships are shown between the constant error and the inverse of the difference between the principal curvatures $\left(R^{2}=.63\right)$ and between the variable error and the inverse of the difference between principal curvatures $\left(R^{2}=.49\right)$. These results suggest that a relative change of curvature could have contributed to the precision with which principal directions were reported.

The findings in Experiment 2 were not entirely consistent with the findings in Experiment 1, where both constant and variable error were homogeneous regardless of curve direction. In Experiment 2, however, responses were worse under the full cue condition for the asymptotic di- 


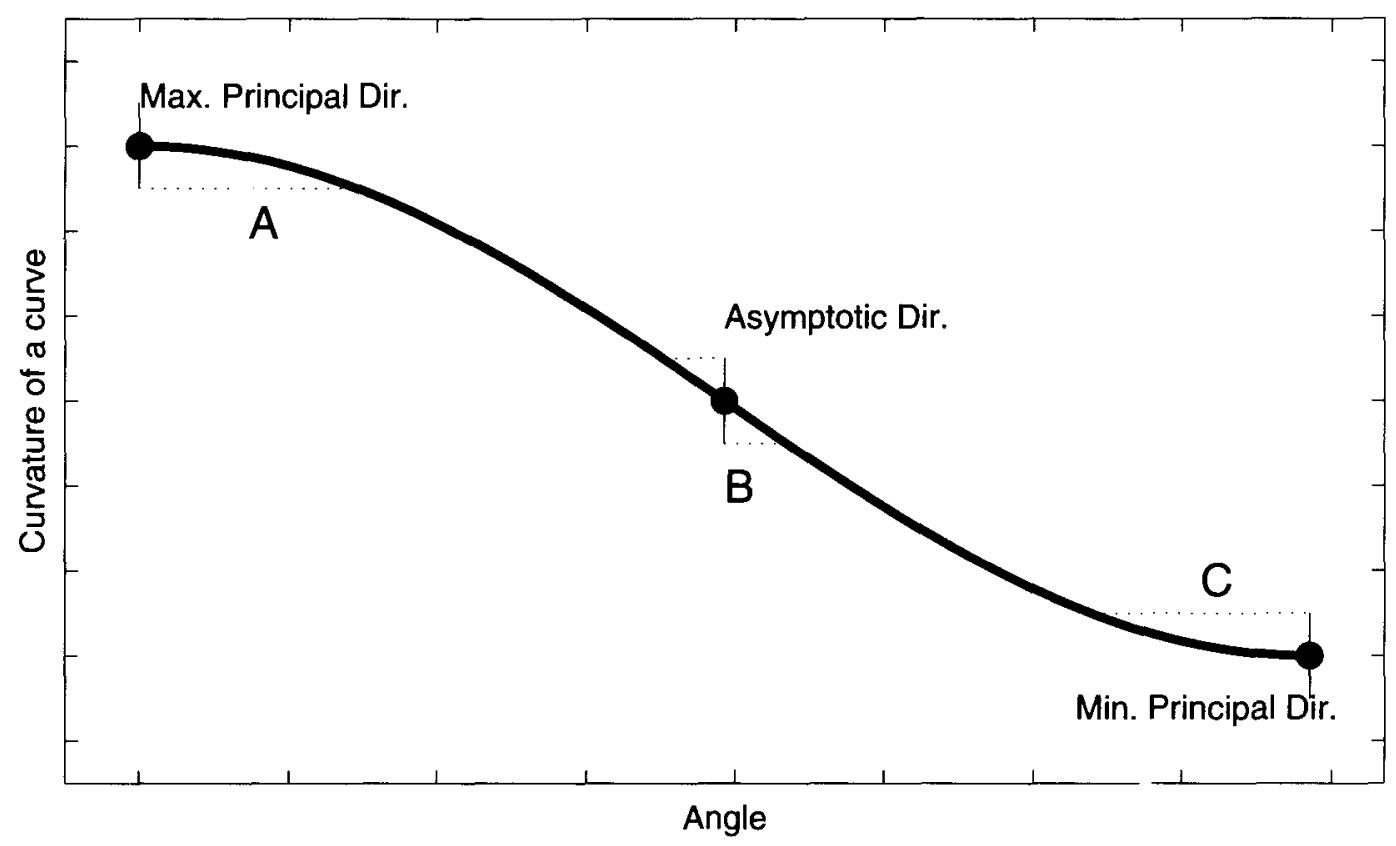

Figure 12. Theoretical prediction of perceived ranges corresponding to the principal directions and the asymptotic direction (see text).

rection than for the principal directions. Therefore, performance depended on the curve direction. How did the subjects report the principal and asymptotic directions during the pointing task? One possibility is that the subjects performed the pointing task on the basis of what value the curvature of curves had in the drawing task. Within this framework, the subjects were assumed to find the direction of probe curvature by comparing the curvatures of curves in various directions.

Such a possibility seems incapable of explaining performance for the principal directions both qualitatively and quantitatively. As Figure 12 shows, regardless of curve direction, the variability of perceived direction should be inversely proportional to the slope of curvature change with respect to direction when the variability of perceived curvature is constant. Thus, if pointing performance for the principal direction task was based on the curvature value indicated by the drawing task, then the error should have been larger for the principal directions than for the asymptotic direction. Direct calculation from the average drawing data of Experiment $1 \mathrm{~B}$ indicates that variability in pointing should have increased from $28^{\circ}$ for the asymptotic direction to $35^{\circ}$ for the principal directions. In Experiment 2 , however, the variability of pointing performance for the principal directions was much smaller $\left(5^{\circ}\right)$ than for the asymptotic direction $\left(22^{\circ}\right)$.

For the asymptotic direction, the variability in pointing performance almost agreed with the prediction from the curvature of curves obtained during the drawing experiment. For the principal directions, however, it is not conceivable that the subjects compared the curvature of curves to find the principal directions. One possible interpretation is that the subjects utilized some global symmetry property of quadratic surfaces. In this experiment, random rotation of the surface and the rectangular aperture eliminated systematic depth information of the edges at the aperture; thus, we think such an interpretation is unlikely. Another interpretation is that the subjects utilized the zero crossing at the change in curvature of curves (derivative with respect to angle $\alpha$, Equation 5); this can be roughly calculated by the third derivative of depth representation. Subjects might possess such a higher order representation of a surface to find the principal direction, and the analysis shown in Figure 11 strongly supports this latter interpretation.

At a minimum, the results of Experiments 1 and 2 suggest that the representation of a surface is different, depending on the task (drawing or pointing), even for the same quadratic surfaces. Our subjects seemed to have utilized the curvature of curves in Experiment 1 and its derivative for principal curvature direction specifically in Experiment 2, while they seemed to have utilized the same representation for the asymptotic direction in Experiment 2.

In Experiment 3, we attempted to directly investigate the existence of task specific representation of a surface. We compared simple perceptual judgments concerning the sign of curvatures and motor responses for the same low curvature of curves by using a psychometric function paradigm. We discuss the difference in the threshold and sensitivity for the same stimuli that are provided by the perceptual and motor tasks. 
Table 6

Results of a Kolmogorov-Smirnov Two-Sample Test Comparing the Psychometric Functions and Raw Data of Perceptual and Motor Tasks

\begin{tabular}{cccc}
\hline Subject & Full & Removed & No Surface \\
\hline H.W. & - & $\mathrm{mP}^{*}$ & - \\
A.K. & - & $\mathrm{mM}^{* *}$ & $\mathrm{mM}^{* *}$ \\
M.E. & $\mathrm{mM}^{* *}$ & $\mathrm{mM}^{* * *}$ & - \\
C.Y. & - & $\mathrm{mP} \dagger$ & $\mathrm{mM}^{*}$ \\
\hline
\end{tabular}

Note-A lowercase $m$ indicates that the result of the motor task was more sensitive. An uppercase M (for motor) or an uppercase P (for perceptual) indicates the more accurate task for judging the sign of the curvature. ${ }^{*} p<.05 .{ }^{* *} p<.01 .{ }^{* *} p<.001 .{ }^{\dagger} p<.1$.

\section{EXPERIMENT 3 Judging the Sign Associated With Curvature of Curves}

In Experiment 3, two issues were addressed. First, we compared sensitivity to the sign of low curvatures for simple perceptual judgments and the motor responses by using a psychometric function paradigm. Second, to demonstrate that our experimental paradigm not only addresses the curvature of curves but also surface curvature, we compared performance for curves embedded in a surface and for the same curves alone.

\section{Method}

Subjects. The subjects were 3 naive subjects (A.K., M.E., and C.Y.), who were paid for their participation, and 1 of the authors (H.W.). All subjects had normal or corrected-to-normal vision.

Apparatus. The same equipment used in Experiment 1 was used in Experiment 3. Additionally, the subjects used a keyboard for responding to the perceptual judgment task.

Stimuli and Conditions. We generated 130 hyperbolic surfaces defined by Equation 2 using the following procedure.

Step 1 . Thirteen probe curves were produced with curvatures around and including the zero curvature. These were $-0.05,-0.04$, $-0.032,-0.024,-0.016,-0.008,0,0.008,0.016,0.024,0.032$, 0.04 , and 0.05 (1/centimeter).

Step 2. Thirteen surfaces were randomly calculated, and each contained a single probe curve. The tip of the probe curve always agreed with the tip of a surface. This procedure was repeated 10 times, so 130 different surfaces were generated.

As visual cue conditions, a full cue condition, a removed cue condition, and a no-surface condition were employed in this experiment. The full cue and removed cue conditions were the same as in Experiments 1 and 2, and the surface stimuli subtended a width of $18.9^{\circ}$ and a length of $24.5^{\circ}$ (degrees of visual arc). A probe curve without disparity was embedded in a surface under the full cue and removed cue conditions. Under the no-surface condition, the surface stimulus was removed, and only a probe curve was presented with disparity. Under all visual cue conditions, a probe curve was simulated by a dimmed and homogeneous red line and a perspective projection. This probe line subtended a width of $0.2^{\circ}$ and a length of $18.5^{\circ}$, and it occupied $0.5 \%$ of the entire display. No shading and texture cues were given for the probe curve.

Tasks. In the perceptual task, the subjects judged the sign associated with the curvature of a probe curve (i.e., convex or concave) and gave their response ("+" or "-") by using a keyboard. Response time was not limited, but the subjects were asked to respond as soon as possible. As a result, the response times for each trial were approximately within $1 \mathrm{sec}$. In the motor task, similar to in Experiment 1 , the subjects drew their response on a probe curve following the beep cues generated by the computer. In both tasks, the probe curves did not disappear during a trial.

Design. Each task consisted of 390 trials (130 probe curvatures $\times 3$ visual cue conditions). The subjects performed both tasks in random order.

Data analysis. In this experiment, we compared data of the two tasks as a ratio of positive responses. From the nominal data for the perceptual task, we calculated the probability with which the subject judged each probe curvature positive. From the data for motor tasks, we extracted drawing trajectory data around the tip of a surface (corresponding to a via point in Experiment 1) by using the same criteria as in Experiment 1. Then the curvatures of extracted trajectories were calculated by using the moving average for bestfitted circles (see the Method section of Experiment 1), and the sign of the curvature was counted for each trajectory.

These converted data were analyzed using a probit-style procedure to obtain the best-fitting cumulative normal distribution for each condition.

\section{Results and Discussion}

Figure 13 shows the estimated psychometric functions for all subjects. A Kolmogorov-Smirnov two-sample test was conducted to look at how a fitted psychometric function can predict raw data obtained under other conditions. Table 6 shows the results of a statistical test for examining whether a given psychometric function is consistent with a particular set of data. From the estimated psychometric function, we calculated the slope at a $50 \%$ threshold and the curvature that corresponds to a $50 \%$ threshold of judgment. We defined sensitivity as the slope value at the $50 \%$ threshold, and we defined accuracy as the inverse of the absolute curvature at the $50 \%$ threshold. In Table 6, a lowercase $m$ indicates that the result of the motor task was more sensitive, and an uppercase $\mathrm{M}$ or $\mathrm{P}$ indicates the more accurate task for judging the sign of curvature.

We found the following: Under the full cue condition, only Subject M.E. showed a significant difference between motor and perceptual tasks. The slope value for M.E. at the $50 \%$ threshold of motor tasks was steeper than that of the perceptual task, and the curvature at the $50 \%$ threshold of motor tasks was closer to zero than that of the perceptual task. This result suggests that she was more accurate and sensitive to the sign of curvature during the motor task than during the perceptual task.

Figure 13 shows that psychometric functions of the subjects were generally flat under the removed cue condition, suggesting that performance was unstable. However, the fitted psychometric functions of motor responses were more sensitive than those of perceptual judgments. Under the no-surface condition, the slope of psychometric functions were steep, and the subjects could sensitively judge the sign of curvature. Subjects A.K. and C.Y. could make a more sensitive and accurate judgment during the motor task under the no-surface condition.

Table 7 summarizes the results of a statistical test that compared the psychometric functions and raw data for possible combinations of visual cue conditions during each task. 

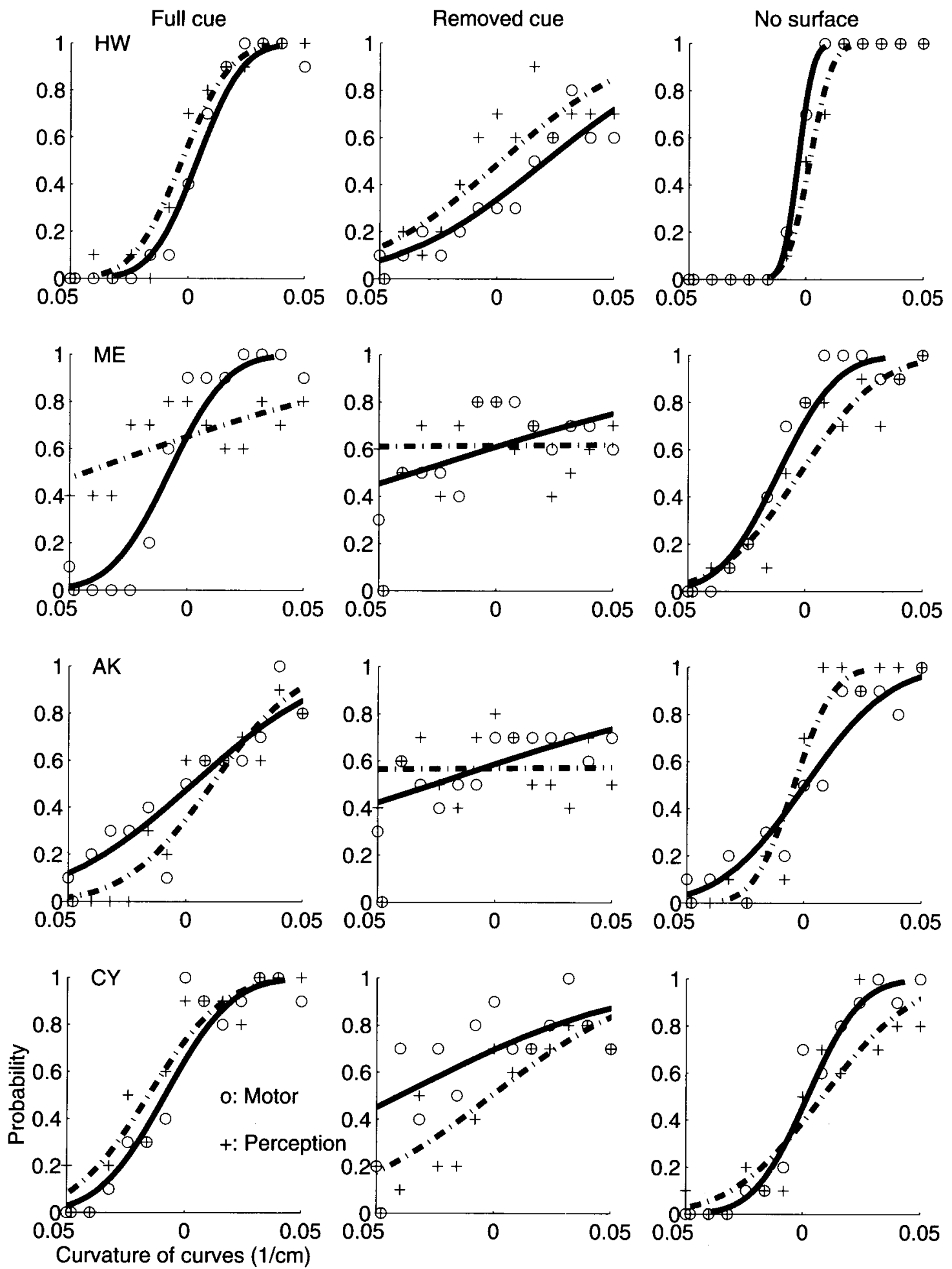

Figure 13. Results of Experiment 3. In each panel, the solid line and the dash-dotted line indicate the estimated psychometric function fitted for the data from the motor task and the perceptual task, respectively. Left, middle, and right columns show the results of full cue surface, removed cue surface, and no-surface conditions, respectively. 
Table 7

Results of a Kolmogorov-Smirnov Two-Sample Test Comparing Psychometric Functions

\begin{tabular}{|c|c|c|c|c|c|c|}
\hline \multirow[b]{2}{*}{ Subject } & \multicolumn{2}{|c|}{ Full vs. Removed } & \multicolumn{2}{|c|}{ Full vs. No-Surface } & \multicolumn{2}{|c|}{ Removed vs. No-Surface } \\
\hline & Motor & Perceptual & Motor & Perceptual & Motor & Perceptual \\
\hline H.W. & * & n.s & * & n.s. & *** & * \\
\hline A.K. & $*$ & $* * *$ & n.s. & $* *$ & $* *$ & $* * *$ \\
\hline M.E. & $* *$ & n.s. & n.s. & ** & $* *$ & $* * *$ \\
\hline C.Y. & $* * *$ & n.s. & n.s. & $* *$ & $* *$ & $\dagger$ \\
\hline
\end{tabular}

Note-n.s., nonsignificant. ${ }^{*} p<.05 .{ }^{* *} p<.01 .{ }^{* * *} p<.001 .{ }^{\dagger} p<.1$.

Although the statistical tests showed differences in the pattern of results between subjects, there were some common features among subjects, summarized as follows: In the motor task, the subjects appeared more sensitive to the curvature of curves for the no-surface condition than for the removed cue condition. Also in the motor task, the subjects appeared more sensitive to the curvature of curves for the full cue condition than for the removed cue condition. In the perceptual judgment task, the subjects appeared more sensitive to the curvature of curves for the no-surface condition than for the removed cue condition.

Our data indicate that the perceived sign of curvature does not always agree with the sign of curvature reported through the use of a motor tasks. When the probe curves were presented alone, the subjects performed differently than in the other visual cue conditions. The fitted psychometric functions had slopes and $50 \%$ thresholds that were more sensitive for the change of curvature. These functions were distinct from those that fit performance in the presence of surfaces. This result suggests that the representations of curves and curves embedded in a surface are different, and this result is consistent with the efforts to understand surface curvature representation, as pursued in this paper.

A final issue in comparisons between the two tasks involves the possible interaction between task and spatial extent. For example, if the motion of the stylus was controlled strictly by matching the local disparity between the stylus and the surface, then the difference between the tasks could be predicted by the fact that the precision of depth order judgments decrease with increasing spatial separation. While this is possible, it is also possible that local motion on the drawing task was dominated by mechanisms related to haptic perception of curvature. Currently though, the literature on haptic discrimination of curvature does not provide precise details as to its spatial sensitivity.

\section{CONCLUSION}

In this paper, we discussed human beings' sensitivity to the amount of surface curvature by using differential geometric techniques and psychophysical experiments. We obtained the following results: (1) Subjects could report principal curvature through a drawing task (Experiment 1B) and could report principal direction through a pointing task (Experiment 2). (2) To a certain extent, subjects could report relative changes in the curvature of curves lying on a surface with the drawing task (Experiment (B). (3) When the data obtained from drawing motions were dealt with as a set of curvatures rather than as a set of depths, Euler's formula was advantageous for predicting data trends (Experiment 1B). (4) The accuracy of reporting surface curvature depends on the difference in principal curvature (umbilicity; Experiments $1 \mathrm{~A}$ and 2. (5) Surface curvature might be dissociated between perceptual processing and motor processing, and the representations associated with the curvature of curves alone and that for curves embedded in a surface are different (Experiment 3). (6) A texture cue influences curvature representation (Experiments 1, 2, and 3).

Interestingly, our results showed that subjects' performance was different depending on the task. For example, sensitivity to the curvature of curves lying on the surface (Experiment 1 ) could not be predicted by performance in the pointing task (Experiment 2). In addition, Experiment 3 showed that the perceived sign of curvature did not always agree with the sign of curvature reported through the use of a motor task. Different patterns of results for the different types of tasks employed in the present series of experiments could have arisen from several possible reasons. One possibility, consistent with neuropsychological data (Goodale \& Milner, 1992; Goodale, Milner, Jakobson, \& Carey, 1991), is that visual processing for visual perceptual tasks and visual processing for visuomotor tasks might involve different streams of processing. On the basis of this, we could expect differences between tasks involving a visual perceptual response and a visuomotor response. Besides differences based on visual and visuomotor processing, it is possible that differences could arise from different task demands on the visual input. For example, it has been suggested from visual perception studies that depth, orientation, and curvature are independently represented (Johnston \& Passmore, 1993; Koenderink et al., 1992). Thus, since each one of these representations can be used to represent a surface, we could expect differences between tasks that stressed different kinds of surface representation.

Given this view that different tasks might stress different perceptual representations and different streams of processing, we can examine the tasks used in the present set of experiments. The tasks used were pointing a direction of curvature, judging the sign of curvature, and draw- 
ing on the surface of a virtual object. Of these tasks, only the task of drawing should be considered an action task. Although pointing is, strictly speaking, a visuomotor task, there was sufficient time for the use of visual feedback, so that the final response was likely determined more by the comparison of two visually perceived directions than the output of any potential action-mediated process. Besides this difference between perception and perception for action, there are no strong reasons to believe that any one of the tasks was intrinsically more or less difficult to perform. Thus, the differences found among the different tasks are probably best considered in terms of the task in conjunction with the visual representation(s) used to perform it.

One could postulate that surface representation may be either qualitative or quantitative (Todd \& Reichel, 1989). Both types of representations have their own strengths and weaknesses. For example, a quantitative representation can produce a description with arbitrary precision but generally requires exhaustive computations and is sensitive to both noise and small changes in viewpoint. A qualitative representation can provide an intrinsic measure of surface structure that is robust to noise and small changes in viewpoint but lacks precision (Todd \& Reichel, 1989). Therefore, we should not conclude that there is only one primary quantitative representation. Humans, however, should have the ability to form the quantitative representation necessary for accomplishing motor behavior requiring accuracy and stability. Furthermore, whether various motor behaviors utilize a general-purpose representation or a task-specific representation is debatable. Depth, surface orientation, and curvature could be transformed into each other by using symbolic manipulation (Johnston \& Passmore, 1993). The possible relationships between visual representation and a task can be summarized as follows: (1) Every task utilizes a common quantitative representation. (2) Depending on the task, the necessary representation is computed by operating on another representation (Carman \& Welch, 1992). (3) The necessary representation is directly encoded from the image intensities (Rogers \& Cagenello, 1989) task specifically.

Our finding in this study suggests a metric coding system for the curvature of curves and relative changes in the curvature of curves. In addition, we have shown the existence of a dissociation between representations of a 3-D surface and a curved line in the 3-D space. This evidence, which suggests the existence of a higher order quantitative surface geometry coded in the human visual system, could be considered as meaningful. As shown in Experiment 1 , the drawing data produced by the subjects could be better predicted when they were treated as a set of curvatures (CFC) rather than as a set of depths (DFF). Moreover, as shown in the Experiment 3, response patterns for the same low curvature were different for a pure perceptual judgment and the drawing task. These results suggest that a specific representation of curvature possibly exists. The issue of the coding process for such a higher order surface geometry still remains unclear. An experimental paradigm that makes it possible to discuss an independent system for curvature representation is necessary.

\section{REFERENCES}

BülthofF, H. H., \& MaLlot, H. A. (1988). Integration of depth modules: Stereo and shading. Journal of the Optical Society of America A, 5, 1749-1758.

Carman, G. J., \& Welch, L. (1992). Three-dimensional illusory contours and surface. Nature, 360, $2189-2202$.

DE VRIES, S. C. (1993). Binocular perception of spatial configuration. Doctoral dissertation, University of Utrecht.

de VRies, S. C., Kappers, A. M. L., \& KoenderinK, J. J. (1993). Shape from stereo: A systematic approach using quadratic surfaces. Perception \& Psychophysics, 53, 71-80.

DE VRIES, S. C., KAPPERS, A. M. L., \& KoENDERINK, J. J. (1994). Influence of surface attitude and curvature scaling on discrimination of binocularly presented curved surfaces. Vision Research, 34, 2409-2423.

Erens, R. G. F., Kappers, A. M. L., \& Koenderink, J. J. (1993). Perception of local shape from shading. Perception \& Psychophysics, 54, 145-156.

GiBson, J. J. (1979). The ecological approach to visual perception. Boston: Houghton Mifflin.

Goodale, M. A., \& Milner, A. D. (1992). Separate visual pathways for perception and action. Trends in Neurosciences, 15, 20-25.

Goodale, M. A., Milner, A. D., Jakobson, L. S., \& Carey, D. P. (1991). A neurological dissociation between perceiving objects and grasping them. Nature, 349, 154-156.

Green, D. M., \& Swets, J. A. (1966). Signal detection theory and psychophysics. New York: Wiley.

HorN, B. K. P. (1986). Robot vision. Cambridge, MA: MIT Press. New York: McGraw-Hill.

HoRN, B. K. P. (1987). Closed-form solution of absolute orientation using unit quarternions. Journal of the Optical Society of America A, 4, 629-642.

Johnston, A., \& PAssmore, P. (1993). Independent encoding of surface orientation and surface curvature. Vision Research, 34, 3005-3012.

KAPPERS, A. M. L., KoENDERINK, J. J., \& LiCHTENEGGer, I. (1994). Haptic identification of curved surfaces. Perception \& Psychophysics, 56, 53-61.

KoEnderink, J. J. (1990). Solid shape, Cambridge, MA: MIT Press.

KoEnderink, J. J., \& VAN DoORN, A. J. (1991). Affine structure from motion. Journal of the Optical Society of America A, 8, 377-385.

KoENDERINK, J. J., vaN DoORN, A. J., \& KAPPERS, A. M. L. (1992). Surface perception in pictures. Perception \& Psychophysics, 52, 487-496.

LIPSHUTZ, M. M. (1969). Theory and problems of differential geometry. New York: McGraw-Hill.

Mingolla, E., \& TodD, J. T. (1986). Perception of solid shape from shading. Biological Cybernetics, 53, 137-151.

Norman, J. F., \& LAPPIN, J. S. (1992). The detection of surface curvatures defined by optical motion. Perception \& Psychophysics, 51, 386-396.

Norman, J. F., TodD, J. T., \& Phillips, F. (1995). The perception of surface orientation from multiple sources of optical information. Perception \& Psychophysics, 57, 629-636.

O'NeILL, B. (1966). Elementary differential geometry. New York: Academic Press.

PhILlips, F., \& TodD, J. T. (1996). Perception of local three-dimensional shape. Journal of Experimental Psychology: Human Perception \& Performance, 22, 930-944.

Pollick, F. E., Nishida, S., Kolke, Y., \& Kawato, M. (1994). Perceived motion in structure from motion: Pointing responses to the axis of rotation. Perception \& Psychophysics, 56, 91-109.

Pollick, F. E., Watanabe, H., \& Kawato, M. (1996). Perception of local orientation from shaded images. Perception \& Psychophysics, 58, 762-780.

Rogers, B., \& CAGEnello, R. (1989). Disparity curvature and the perception of three-dimensional surfaces. Nature, 338, 135-137. 
SchWARTZ, A. B. (1994). Direct cortical representation of drawing. Science, 265, 540-542.

STEVENS, K. A. (1981). The visual interpretation of surface contours. Artificial Intelligence, 17, 47-43.

ToDD, J. T., \& ReICHEL, F. D. (1989). Ordinal structure in the visual perception and cognition of smoothly curved surface. Psychological Review, 96, 643-657.

VAN DAMME, W. J. M., \& VAN DE GRIND, W. A. (1993). Active vision and the identification of three-dimensional shape. Vision Research, 33, 1581-1587.

\section{NOTES}

1. The three probe points do not have disparity, but the surface surrounding them has disparity information. Therefore, subjects could use the depth information in the vicinity of those points.

2. The coefficient of determination $\left(R^{2}\right)$ is defined as the square of the multiple correlation coefficient between the reported curvature of normal section and the predicted curvature of normal section reconstructed by Euler's formula.

\section{APPENDIX \\ Calculating the Perceived Surface \\ Curvature From the Drawing Data}

\section{General Knowledge About Curvature \\ From Curves Lying on a Surface}

The normal section of a surface is the intersection of a smooth surface $S$ and the plane containing the surface normal $N$ at any given point $P_{0}$ (see Figure 1). The maximum and minimum curvatures of a normal section are called the maximum principal curvature $\left(\kappa_{\max }\right)$ and the minimum principal curvature $\left(\kappa_{\min }\right)$. The corresponding directions of these principal curvatures are called the principal directions, and these two principal directions are always orthogonal.

For any curve passing through the common point $P_{0}$ (via point) and lying on the surface, let $\boldsymbol{\kappa}^{i}$ denote the curvature of $C^{i}$ at $P_{0}$ (in our analysis, we had three repetitions of four directions, resulting in 12 curves $\left.\left[C^{i}, i=1, \ldots, 12\right]\right)$. It is known that the curvature $\kappa_{S}^{i}$ of the slanted section, which is the inter-

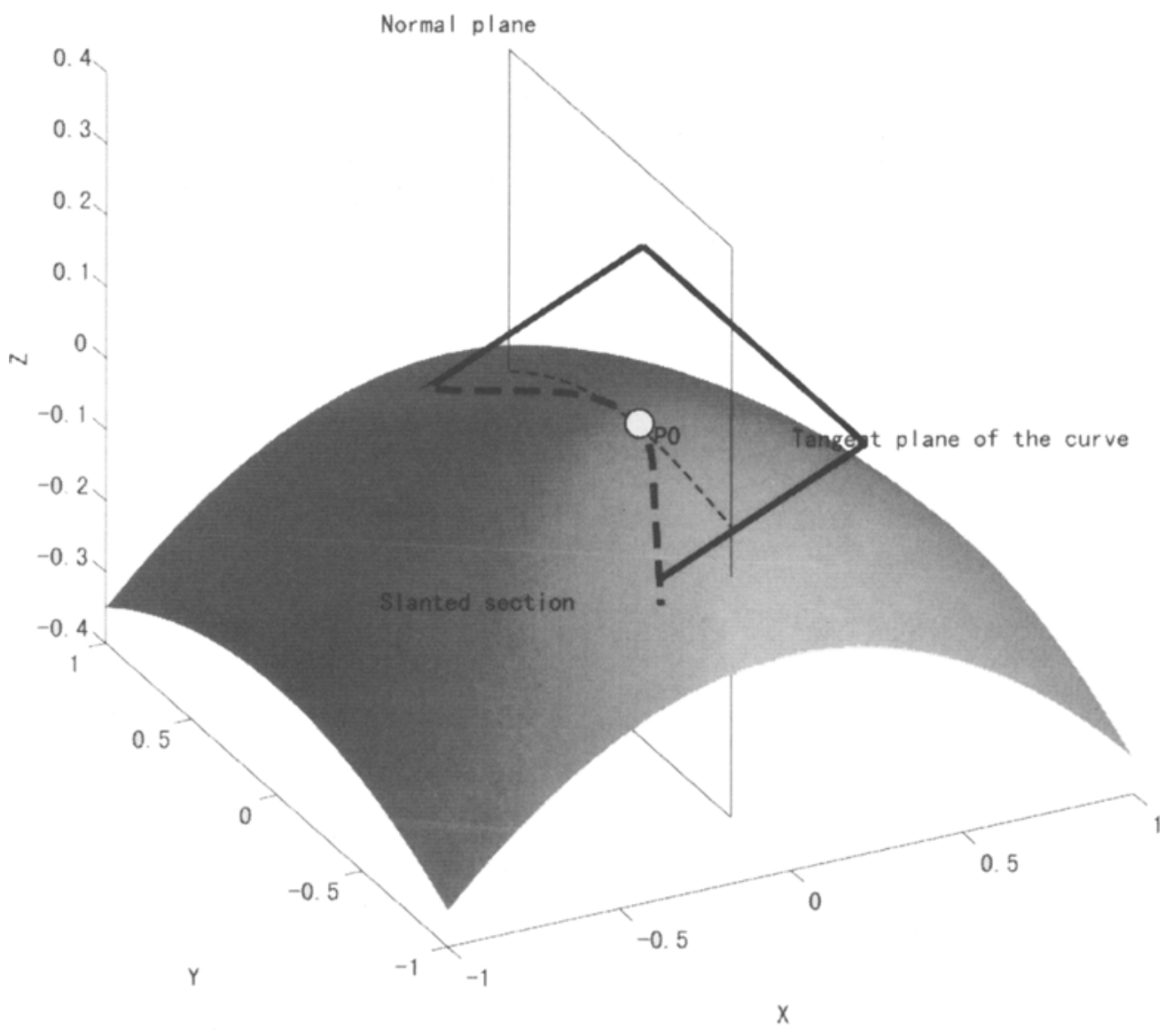

Figure A1. Surface, tangent plane of the curve, and slanted section. When the curve passing through $\boldsymbol{P}_{0}$ on the surface is drawn freely, the curvature of the curve at $P_{0}$ is equal to the curvature of the slanted section. The relationship between the normal curvature and the curvature of the tangent plane of the curve containing the slanted section can be described as a function of the angle between normal plane and tangent plane of the curve (Meusnier's lemma). 


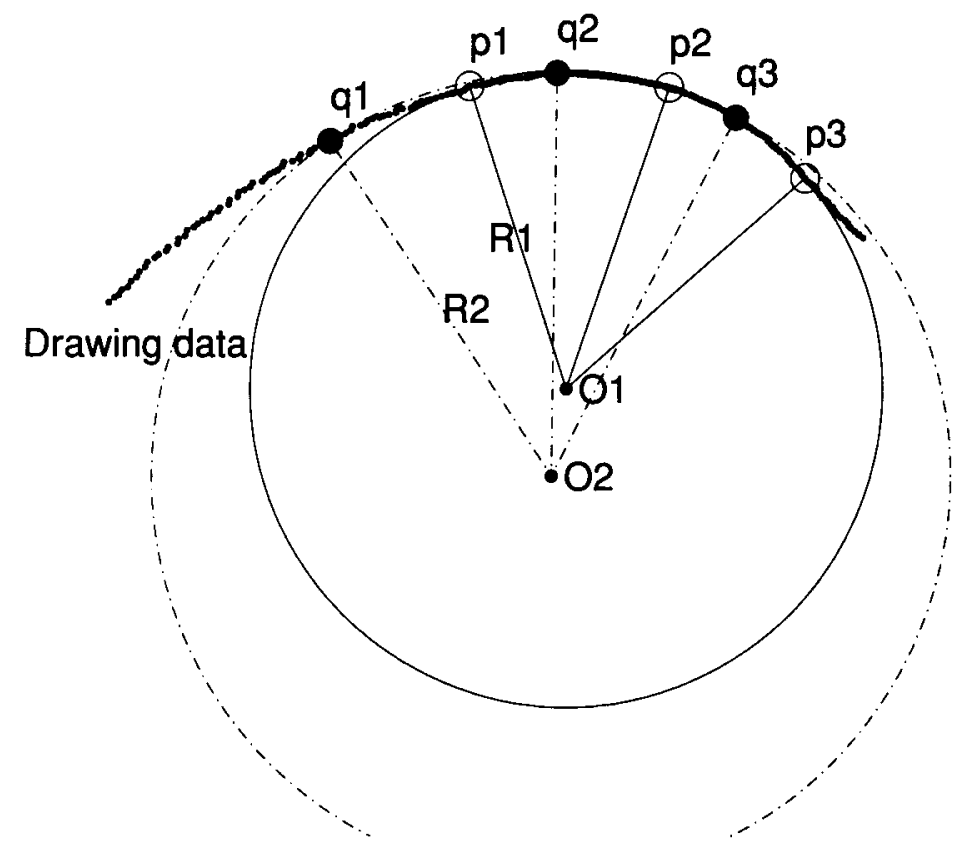

Figure A2. Moving average of fitted circle. To calculate the curvature of each drawing trajectory, which consists of 201 sampling points, three points at 50point intervals are picked (p1, p2, p3), and the circle with a radius of R1 is nitted for those three points (O1). The same procedure is applied for the other set of three points (q1,q2, q3), and the circle $O 2$ with a radius of $R 2$ is found. Thus, we have a set of 101 fitted circles, and their average radius is defined as the radius of curvature of the drawing trajectory.

section of the surface and the tangent plane of the curve, is equal to the curvature $\kappa^{i}$ of the curve at $P_{0}$ :

$$
\boldsymbol{\kappa}_{S}^{i}=\boldsymbol{\kappa}^{i}
$$

Let $\theta$ denote the angle between the normal vector $\mathrm{N}$ of the surface at $P_{0}$ and the tangent plane of the curve $C^{i}$. Then, between the curvature $\kappa_{S}^{i}$ of curves and the curvature $\kappa_{N}^{i}$ of a normal section, the following equation holds (Meusnier's lemma; Figure Al):

$$
\kappa_{N}^{i}=\kappa_{S}^{i} \cos \theta
$$

For each normal section, the curvature $\boldsymbol{\kappa}_{N}^{i}$ is given by Euler's formula (Equation 1). A combination of Euler's formula and Meusnier's lemma (Equation A2), produces

$$
\kappa_{S}^{i} \cos \theta=\kappa_{\max } \cos ^{2} \alpha^{i}+\kappa_{\min } \sin ^{2} \alpha^{i} .
$$

Angles of direction of maximum curvature (principal direction) and the tangent line of curve $C^{i}$ are measured by an arbitrary 2-D coordinate system attached to the tangent plane of the surface at $P_{0} . \theta_{M}$ and $\alpha$ denote the principal direction and the angle of the tangent line of the curve, respectively. Therefore, the following equation holds:

$$
\kappa_{S}^{i} \cos \theta=\kappa_{\max } \cos ^{2}\left(\alpha^{i}-\theta_{M}\right)+\kappa_{\min } \sin ^{2}\left(\alpha^{i}-\theta_{M}\right) .
$$

\section{Curvature From Curves (CFC) Method}

Here, we assume that there exists a plane and a circle contained in it that well approximates a set of data points lying on each curve $C^{i}$. This plane approximates to a tangent plane of the curve that contains the slanted section.

The equation for the circle in the plane in 3-D space can be written in vector notation as follows:

$$
\begin{aligned}
\left\|e_{c}\right\| & =1 \\
\left(r-r_{c}\right) e_{c} & =0 \\
\left\|r-r_{c}\right\| & =R a d .
\end{aligned}
$$

Here, $e_{c}, r, r_{c}$, and $R a d$ are the normal vector of the plane, the position on the circle, the position of the center of the circle, and the radius of the circle, respectively. Let $r_{j},(j=1,2, \ldots$, 201 ) be a set of discrete data points on a single curve trajectory generated by a subject. We pick three points at 50 -point intervals from a single curve trajectory and have 101 sets of three points. Then, we fit a circle that satisfies criteria (Equation A5) for each set of three points. The curvature of $C^{i}(=1 / R a d)$, the two angles $\gamma^{i}$ (between the surface normal $\mathrm{N}$ and the tangent plane containing curve $C^{i}$ ) and $\theta^{i}$ (between the direction of maximum curvature and the tangent line of the $i$ th curve) are obtained by averaging 101 fitted circles (Figure A2). From Meusnier's lemma (Equation A2), we can calculate the curvature of the $i$ th normal section, $\boldsymbol{\kappa}_{N}^{i}$.

From Equation A4 (the general version combining Meusnier's lemma and Euler's formula), we obtain the following:

$$
\kappa_{S}^{i} \cos \gamma=\mathrm{A}+\mathrm{B} \cos ^{2} \alpha^{i}+\mathrm{C} \sin ^{2} \alpha^{i} .
$$

Here, $A, B$, and $C$ are

$$
A=\frac{\kappa_{\max }+\kappa_{\min }}{2}
$$




$$
\begin{aligned}
& B=\frac{\kappa_{\max }-\kappa_{\min }}{2} \cos 2 \theta_{M}, \\
& C=\frac{\kappa_{\max }-\kappa_{\min }}{2} \sin 2 \theta_{M} .
\end{aligned}
$$

From the three repetitions in four directions, we can obtain 12 equations involving $A, B$, and $C$. The solution to such a system of equations can be recovered by, for example, the MoorePenrose pseudo-inverse matrix. From this, the principal curvatures and angle are obtained as follows:

$$
\begin{aligned}
\kappa_{\max } & =A+\sqrt{B^{2}+C^{2}} \\
\kappa_{\min } & =A-\sqrt{B^{2}+C^{2}} \\
\theta_{M} & =\frac{1}{2} \arctan \frac{C}{B}
\end{aligned}
$$

\section{Differentiation From}

\section{Best-Fitted Surface (DFF) Method}

In this section, we introduce another method for estimating surface curvature from curves lying on a. surface. We can calculate the same differential geometrical surface properties as with the CFC method, but now we assume that subjects are reporting surface depth with their drawing motions, and we can fit the following second-order polynomial surface to the drawing trajectories by a least mean square error criterion:

$$
f(x, y)=a x^{2}+b y^{2}+c x y+d x+e y+f .
$$

It is known that, for a surface expressed in its explicit form, the principal curvature $\kappa_{\max }$ and $\kappa_{\min }$ can be obtained as the inverse solutions of the following quadratic equation:

$$
\left(u w-v^{2}\right) \rho^{2}+h\left[2 s t v-\left(1+s^{2}\right) w-\left(1+t^{2} u\right)\right] \rho+h^{4}=0
$$

Here,

$$
\begin{aligned}
& s=f_{x}, t=f_{y}, u=f_{x x}, \\
& v=f_{x y}, w=f_{y y}, h=\sqrt{1+s^{2}+t^{2}} .
\end{aligned}
$$

The solutions $\rho$ of Equation A10 are called the radii of principal curvatures. The principal directions are obtained from the solution $d y / d x$ of the following equation:

$$
\begin{gathered}
{\left[s t w-v\left(1+t^{2}\right)\right]\left(\frac{d y}{d x}\right)^{2}+\left[w\left(1+s^{2}\right)-u\left(1+t^{2}\right)\right]\left(\frac{d y}{d x}\right)} \\
+\left[v\left(1+s^{2}\right)-s t u\right]=0
\end{gathered}
$$

(Manuscript received June 5, 1997; revision accepted for publication July 12, 1998.) 